\title{
SECOND ORDER FULLY DISCRETE ENERGY STABLE METHODS ON STAGGERED GRIDS FOR HYDRODYNAMIC PHASE FIELD MODELS OF BINARY VISCOUS FLUIDS*
}

\author{
YUEZHENG GONG ${ }^{\dagger}$, JIA ZHAO ${ }^{\ddagger}$, AND QI WANG§
}

\begin{abstract}
We present second order, fully discrete, energy stable methods on spatially staggered grids for a hydrodynamic phase field model of binary viscous fluid mixtures in a confined geometry subject to both physical and periodic boundary conditions. We apply the energy quadratization strategy to develop a linear-implicit scheme. We then extend it to a decoupled, linear scheme by introducing an intermediate velocity term so that the phase variable, velocity field, and pressure can be solved sequentially. The two new, fully discrete linear schemes are then shown to be unconditionally energy stable, and the linear systems resulting from the schemes are proved uniquely solvable. Rates of convergence of the two linear schemes in both space and time are verified numerically. The decoupled scheme tends to introduce excessive dissipation compared to the coupled one. The coupled scheme is then used to simulate fluid drops of one fluid in the matrix of another fluid as well as mixing dynamics of binary polymeric, viscous solutions. The numerical results in mixing dynamics reveals the dramatic difference between the morphology in the simulations obtained using the two different boundary conditions (physical vs. periodic), demonstrating the importance of using proper boundary conditions in fluid dynamics simulations.
\end{abstract}

Key words. energy quadratization, fully discrete energy stable scheme, staggered grids, finite difference methods

AMS subject classifications. $65 \mathrm{M}, 76 \mathrm{~T}$

DOI. $10.1137 / 17 \mathrm{M} 1135451$

1. Introduction. Multiphase fluid flows exist ubiquitously in nature and in industrial processes. One of the useful models to describe hydrodynamics of multiphase fluid flow is the diffuse interface model, also known as the phase field model. The phase field method resolves the material's interface by capturing it implicitly instead of tracking it explicitly. When the phase field method is applied to study immiscible fluid mixtures, a smooth phase variable is introduced [5] whose spatially varying transitional layer represents the interface. Due to its simplicity in the theoretical formulation and numerical implementation, the phase field method has been widely used in fields where multiple material phases are involved. These include life sciences (cell biology [28, 52, 53, 43], biofilms [51, 50, 54], cell adhesion and motility $[32,28,27,38,30]$, cell membrane $[40,8,9,11])$, materials science $[6,39]$, fluid

\footnotetext{
*Submitted to the journal's Computational Methods in Science and Engineering section June 20, 2017; accepted for publication (in revised form) January 5, 2018; published electronically April 5, 2018.

http://www.siam.org/journals/sisc/40-2/M113545.html

Funding: The first author's work was partially supported by the China Postdoctoral Science Foundation through grant 2016M591054 and by the foundation of Jiangsu Key Laboratory for Numerical Simulation of Large Scale Complex Systems (201703). The second author's work was partially supported by a startup fund and a Research Catalyst Grant from the Office of Research and Graduate Studies at USU. The third author's work was partially supported by award DMS-1517347 and by NSFC awards 11571032, 91630207, and NSAF-U1530401.

${ }^{\dagger}$ College of Science, Nanjing University of Aeronautics and Astronautics, Nanjing 210016, China (gongyuezheng@nuaa.edu.cn).

${ }^{\ddagger}$ Department of Mathematics \& Statistics, Utah State University, Logan, UT 84322 (jia.zhao@ usu.edu).

$\S$ Corresponding author. School of Materials and Engineering, Nankai University, Tianjin 300084, China; Department of Mathematics, University of South Carolina, Columbia, SC 29208; and Beijing Computational Science Research Center, Beijing 100084, China (qwang@math.sc.edu).
}

B528 
dynamics [29], and image processing [3, 4].

To model an incompressible binary fluid mixture consisting of two fluids - say fluid $A$ and fluid $B$-using the phase field approach, one introduces a phase variable $\phi$ to represent the volume fraction of fluid $A$ such that the volume fraction of fluid $B$ is represented by $1-\phi$. A free energy $F$ has to be developed to account for the material property of the two fluids, which is given by a functional of the phase variable. Then the phase transport equation, together with the hydrodynamic equations, has to be derived in a thermodynamically consistent fashion to obey the second law of thermodynamics $[29,37,25,19]$. A hydrodynamic phase field model for an incompressible binary fluid mixture has been derived by various authors, consisting of the following equations $[23,26]$ :

$$
\left\{\begin{array}{l}
\rho\left(\partial_{t} \mathbf{v}+\mathbf{v} \cdot \nabla \mathbf{v}\right)=-\nabla p+\eta \Delta \mathbf{v}-\phi \nabla \mu \\
\nabla \cdot \mathbf{v}=0 \\
\partial_{t} \phi+\nabla \cdot(\phi \mathbf{v})=M \Delta \mu
\end{array}\right.
$$

where $\rho$ is the mass density of the mixture (a constant), $\mathbf{v}$ is the mass-average velocity, $p$ is the hydrostatic pressure, $M$ is the mobility coefficient (assuming it is a constant), and $\mu=\frac{\delta F}{\delta \phi}$ is the chemical potential. The suitable boundary conditions for the governing system of equations include periodic boundary conditions or the following physical boundary conditions:

$$
\left.\mathbf{v}\right|_{\partial \Omega}=\mathbf{0},\left.\quad \nabla \phi \cdot \mathbf{n}\right|_{\partial \Omega}=0,\left.\quad \nabla \mu \cdot \mathbf{n}\right|_{\partial \Omega}=0 .
$$

Strictly speaking, this model is valid only for the binary fluid mixture in which the two fluid components are of identical mass density! When the densities are different, this is an approximation of the correct model, known as the quasi-incompressible multiphase fluid model $[29,25,1]$. So, users should be aware of its applicability when applying it.

Model (1.1) has been used to study incompressible binary fluid mixtures, in which the free energy $F[\phi]=\int_{\Omega}\left(\frac{\gamma_{1}}{2}|\nabla \phi|^{2}+f(\phi)\right) d \mathbf{x}$ is adopted, where $\Omega$ is the domain that the fluid occupies, $\gamma_{1}$ is a parameter measuring the strength of the conformational entropy, and $f(\phi)$ is the bulk energy density. For immiscible binary fluids, one choice of the bulk energy density is the double-well potential $f(\phi)=\gamma_{2} \phi^{2}(1-\phi)^{2}$, where $\gamma_{2}$ measures the strength of the repulsive potential. In the sharp-interface limit, $\sqrt{\gamma_{1} \gamma_{2}}$ is proportional to the surface tension, and $\sqrt{\frac{\gamma_{1}}{\gamma_{2}}}$ controls the interfacial thickness. For miscible binary viscous polymeric blends, $f(\phi)$ can be the Flory-Huggins free energy density $f(\phi)=\gamma_{2}\left(\frac{\phi}{N_{1}} \ln \phi+\frac{(1-\phi)}{N_{2}} \ln (1-\phi)+\chi \phi(1-\phi)\right)$, where $N_{1}$ and $N_{2}$ are the polymerization index for the $A$ and $B$ phase, respectively, $\chi$ is the mixing parameter, and $\gamma_{2}$ measures the strength of the bulk potential.

The hydrodynamic phase field model given by (1.1) is thermodynamically consistent with the second law of thermodynamics and respects an energy dissipation property (see [14], for instance). Given the dissipative property of the governing system of equations, one would like any numerical schemes developed for the system to respect an analogous energy dissipation law at the discrete level. A numerical scheme of this property is known as an energy stable scheme.

Recently, based on the original idea of Badia, Guillen-Gonzalez, and GutierrezSantacreu [2] in the treatment of liquid crystal models and Guillen-Gonzalez and Tierra [17], we, together with Xiaofeng Yang, proposed a new strategy and coined it the (invariant) energy quadratization (EQ or IEQ) method for thermodynamic models 
$[45,44,46,48,55,47]$. See [57] for a detailed review. We note that this is a general technique that can be applied to nonequilibrium thermodynamic systems so long as an energy dissipation law exists or, equivalently, the positive entropy production property is demonstrated. The idea is to transform the free energy into a quadratic form by introducing intermediate variables. The time evolution of the intermediate variables are space-independent, i.e., they are ordinary differential equations in time. This method allows one to develop linear energy stable schemes that respect the energy dissipation law. In a sequence of recent papers, the idea of EQ has been applied to a host of hydrodynamic models $[56,15,21,14]$. Notice that the EQ method is for developing semidiscrete schemes in time; thus, the spatial discretization is decoupled from the EQ method, leaving a large degree of freedom to develop proper spatial discretizations.

In this paper, we develop efficient numerical schemes for the hydrodynamic phase field model (1.1) in a confined geometry subject to physical as well as periodic boundary conditions. We combine the EQ strategy in time with a finite difference discretization in space on staggered grids to develop linear, energy stable schemes that respect the energy dissipation law. Specifically, we propose a second order spatial discretization to discretize the hydrodynamic phase field model (1.1) on staggered grids in space, arriving at a system of time-dependent differential-algebraic equations (DAEs). Then we bring on the EQ strategy to reformulate the model into an equivalent one by introducing new (intermediate) variables. The reformulated model allows us to design linear schemes to achieve second order accuracy in time. Afterward, a decoupling strategy $[35,36,58,49]$ is brought in to obtain a linearly decoupled scheme such that the velocity field, phase variable, and pressure can be solved sequentially. The governing equation for each of the physical variables is an elliptic-type equation on which fast and efficient solvers can be applied. The novelty of this paper is that we present a systematic approach to develop fully discrete, second order, linear schemes and show the unique solvability of the linear systems at each time step. Even though there are several existing works on fully discrete schemes for the hydrodynamic phase field model or its simplified versions [41, 7, 20,22], all of them are either only first order in time or nonlinear. In comparison, our linear schemes can be more efficient in implementation and solution procedures. We remark that some second order (linear) energy stable schemes have been developed for thermodynamic phase field equations in recently years $[12,24,34,18,10]$, which may potentially be applicable to hydrodynamic phase field models (although this hasn't been attempted yet). The schemes are presented in $2 \mathrm{D}$ in this paper for the sake of simplicity, but they can be readily extended to 3D. In fact, some of the numerical results are given in 3D space near the end of the article.

In the rest of the paper, we first define notation and give some useful lemmas in section 2 . We present the second order spatial discretization in section 3 and the fully discrete schemes subsequently in section 4. Afterward, we show that the two linear schemes are uniquely solvable in section 5 . Then numerical convergence tests are carried out in section 6 together with two numerical simulation results with respect to two applications. Finally, we give the conclusion in the last section.

2. Notation and some useful lemmas. To simplify the presentation, we first introduce some notation and useful lemmas. Following the notation in $[41,33,42,7]$, we denote $\Omega=\left[0, L_{x}\right] \times\left[0, L_{y}\right]$ as the computational domain, where $L_{x}$ and $L_{y}$ are two positive numbers. We divide the domain into rectangular meshes with mesh size $h_{x}=L_{x} / N_{x}, h_{y}=L_{y} / N_{y}$, where $N_{x}$ and $N_{y}$ are two positive integers. We define the 
following 1D sets for grid points:

$$
\begin{aligned}
& E_{x}=\left\{x_{i+\frac{1}{2}} \mid i=0,1, \ldots, N_{x}\right\}, C_{x}=\left\{x_{i} \mid i=1,2, \ldots, N_{x}\right\}, C_{\bar{x}}=\left\{x_{i} \mid i=0,1, \ldots, N_{x}+1\right\}, \\
& E_{y}=\left\{y_{j+\frac{1}{2}} \mid j=0,1, \ldots, N_{y}\right\}, C_{y}=\left\{y_{j} \mid j=1,2, \ldots, N_{y}\right\}, C_{\bar{y}}=\left\{y_{j} \mid j=0,1, \ldots, N_{y}+1\right\},
\end{aligned}
$$

where $x_{l}=\left(l-\frac{1}{2}\right) h_{x}, y_{l}=\left(l-\frac{1}{2}\right) h_{y}$, and $l$ can take on either integer or half-integer values. $E_{x}$ is called a uniform partition of $\left[0, L_{x}\right]$ of size $N_{x}$, and its elements are called edge-centered points. The elements of $C_{x}$ and $C_{\bar{x}}$ are called cell-centered points. The two points belonging to $C_{\bar{x}} \backslash C_{x}$ are called ghost points. Analogously, the set $E_{y}$ is a uniform partition of $\left[0, L_{y}\right]$ of size $N_{y}$, called edge-centered points, and $C_{y}$ and $C_{\bar{y}}$ contain the cell-centered points of the interval $\left[0, L_{y}\right]$.

We define the following discrete function spaces:

$\mathcal{C}_{x \times y}=\left\{\phi: C_{x} \times C_{y} \rightarrow \mathbb{R}\right\}, \quad \mathcal{C}_{\bar{x} \times y}=\left\{\phi: C_{\bar{x}} \times C_{y} \rightarrow \mathbb{R}\right\}, \quad \mathcal{C}_{x \times \bar{y}}=\left\{\phi: C_{x} \times C_{\bar{y}} \rightarrow \mathbb{R}\right\}$, $\mathcal{C}_{\bar{x} \times \bar{y}}=\left\{\phi: C_{\bar{x}} \times C_{\bar{y}} \rightarrow \mathbb{R}\right\}, \quad \mathcal{E}_{x \times y}^{e w}=\left\{u: E_{x} \times C_{y} \rightarrow \mathbb{R}\right\}, \quad \mathcal{E}_{x \times \bar{y}}^{e w}=\left\{u: E_{x} \times C_{\bar{y}} \rightarrow \mathbb{R}\right\}$, $\mathcal{E}_{x \times y}^{n s}=\left\{v: C_{x} \times E_{y} \rightarrow \mathbb{R}\right\}, \quad \mathcal{E}_{\bar{x} \times y}^{n s}=\left\{v: C_{\bar{x}} \times E_{y} \rightarrow \mathbb{R}\right\}, \quad \mathcal{V}_{x \times y}=\left\{f: E_{x} \times E_{y} \rightarrow \mathbb{R}\right\}$.

We denote the cell-centered, edge-centered, and vertex-centered discrete functions as follows:

cell centered functions: $\phi, \psi, \mu, p, q \in \mathcal{C}_{x \times y} \cup \mathcal{C}_{\bar{x} \times y} \cup \mathcal{C}_{x \times \bar{y}} \cup \mathcal{C}_{\bar{x} \times \bar{y}}$, east west edge centered functions: $u, r \in \mathcal{E}_{x \times y}^{e w} \cup \mathcal{E}_{x \times \bar{y}}^{e w}$, north south edge centered functions: $v, w \in \mathcal{E}_{x \times y}^{n s} \cup \mathcal{E}_{\bar{x} \times y}^{n s}$, vertex centered functions: $f, g \in \mathcal{V}_{x \times y}$.

We define the discrete function spaces with homogeneous Dirichlet boundary conditions as follows:

$$
\begin{aligned}
& \mathcal{E}_{x \times y}^{e w 0}=\left\{u \in \mathcal{E}_{x \times y}^{e w} \cup \mathcal{E}_{x \times \bar{y}}^{e w} \mid u_{\frac{1}{2}, j}=u_{N_{x}+\frac{1}{2}, j}=0, j=1,2, \ldots, N_{y}\right\}, \\
& \mathcal{E}_{x \times y}^{n s 0}=\left\{v \in \mathcal{E}_{x \times y}^{n s} \cup \mathcal{E}_{\bar{x} \times y}^{n s} \mid v_{i, \frac{1}{2}}=v_{i, N_{y}+\frac{1}{2}}=0, i=1,2, \ldots, N_{x}\right\}, \\
& \mathcal{V}_{x \times y}^{0}=\left\{f \in \mathcal{V}_{x \times y} \mid f_{\frac{1}{2}, j+\frac{1}{2}}=f_{N_{x}+\frac{1}{2}, j+\frac{1}{2}}=f_{i+\frac{1}{2}, \frac{1}{2}}=f_{i+\frac{1}{2}, N_{y}+\frac{1}{2}}=0,\right. \\
&\left.i=0,1, \ldots, N_{x}, j=0,1, \ldots, N_{y}\right\} .
\end{aligned}
$$

We define the east-west-edge-to-center average and difference operator as $a_{x}, d_{x}$ : $\mathcal{E}_{x \times \bar{y}}^{e w} \cup \mathcal{V}_{x \times y} \rightarrow \mathcal{C}_{x \times \bar{y}} \cup \mathcal{E}_{x \times y}^{n s}$ in componentwise forms:

$$
\begin{gathered}
a_{x} u_{i, j}=\frac{1}{2}\left(u_{i+\frac{1}{2}, j}+u_{i-\frac{1}{2}, j}\right), \quad d_{x} u_{i, j}=\frac{1}{h_{x}}\left(u_{i+\frac{1}{2}, j}-u_{i-\frac{1}{2}, j}\right), \quad a_{x} u, d_{x} u \in \mathcal{C}_{x \times \bar{y}}, \\
a_{x} f_{i, j+\frac{1}{2}}=\frac{1}{2}\left(f_{i+\frac{1}{2}, j+\frac{1}{2}}+f_{i-\frac{1}{2}, j+\frac{1}{2}}\right), \quad d_{x} f_{i, j+\frac{1}{2}}=\frac{1}{h_{x}}\left(f_{i+\frac{1}{2}, j+\frac{1}{2}}-f_{i-\frac{1}{2}, j+\frac{1}{2}}\right), \\
a_{x} f, d_{x} f \in \mathcal{E}_{x \times y}^{n s} .
\end{gathered}
$$

The north-south-edge-to-center average and difference operators are defined as $a_{y}, d_{y}$ : $\mathcal{E}_{\bar{x} \times y}^{n s} \cup \mathcal{V}_{x \times y} \rightarrow \mathcal{C}_{\bar{x} \times y} \cup \mathcal{E}_{x \times y}^{e w}$ in componentwise forms:

$$
\begin{array}{r}
a_{y} v_{i, j}=\frac{1}{2}\left(v_{i, j+\frac{1}{2}}+v_{i, j-\frac{1}{2}}\right), \quad d_{y} v_{i, j}=\frac{1}{h_{y}}\left(v_{i, j+\frac{1}{2}}-v_{i, j-\frac{1}{2}}\right), \quad a_{y} v, d_{y} v \in \mathcal{C}_{\bar{x} \times y}, \\
a_{y} f_{i+\frac{1}{2}, j}=\frac{1}{2}\left(f_{i+\frac{1}{2}, j+\frac{1}{2}}+f_{i+\frac{1}{2}, j-\frac{1}{2}}\right), \quad d_{y} f_{i+\frac{1}{2}, j}=\frac{1}{h_{y}}\left(f_{i+\frac{1}{2}, j+\frac{1}{2}}-f_{i+\frac{1}{2}, j-\frac{1}{2}}\right), \\
a_{y} f, d_{y} f \in \mathcal{E}_{x \times y}^{e w} .
\end{array}
$$

Copyright (c) by SIAM. Unauthorized reproduction of this article is prohibited. 
The center-to-east-west-edge average and difference operators are defined as $A_{x}, D_{x}$ : $\mathcal{C}_{\bar{x} \times \bar{y}} \cup \mathcal{E}_{\bar{x} \times y}^{n s} \rightarrow \mathcal{E}_{x \times \bar{y}}^{e w} \cup \mathcal{V}_{x \times y}$ in componentwise forms:

$$
\begin{array}{r}
A_{x} \phi_{i+\frac{1}{2}, j}=\frac{1}{2}\left(\phi_{i+1, j}+\phi_{i, j}\right), \quad D_{x} \phi_{i+\frac{1}{2}, j}=\frac{1}{h_{x}}\left(\phi_{i+1, j}-\phi_{i, j}\right), \quad A_{x} \phi, D_{x} \phi \in \mathcal{E}_{x \times \bar{y}}^{e w}, \\
A_{x} v_{i+\frac{1}{2}, j+\frac{1}{2}}=\frac{1}{2}\left(v_{i+1, j+\frac{1}{2}}+v_{i, j+\frac{1}{2}}\right), \quad D_{x} v_{i+\frac{1}{2}, j+\frac{1}{2}}=\frac{1}{h_{x}}\left(v_{i+1, j+\frac{1}{2}}-v_{i, j+\frac{1}{2}}\right), \\
A_{x} v, D_{x} v \in \mathcal{V}_{x \times y} .
\end{array}
$$

The center-to-north-south-edge average and difference operators are defined as $A_{y}, D_{y}$ : $\mathcal{C}_{\bar{x} \times \bar{y}} \cup \mathcal{E}_{x \times \bar{y}}^{e w} \rightarrow \mathcal{E}_{\bar{x} \times y}^{n s} \cup \mathcal{V}_{x \times y}$ in componentwise forms:

$$
\begin{array}{r}
A_{y} \phi_{i, j+\frac{1}{2}}=\frac{1}{2}\left(\phi_{i, j+1}+\phi_{i, j}\right), \quad D_{y} \phi_{i, j+\frac{1}{2}}=\frac{1}{h_{y}}\left(\phi_{i, j+1}-\phi_{i, j}\right), \quad A_{y} \phi, D_{y} \phi \in \mathcal{E}_{\bar{x} \times y}^{n s}, \\
A_{y} u_{i+\frac{1}{2}, j+\frac{1}{2}}=\frac{1}{2}\left(u_{i+\frac{1}{2}, j+1}+u_{i+\frac{1}{2}, j}\right), \quad D_{y} u_{i+\frac{1}{2}, j+\frac{1}{2}}=\frac{1}{h_{y}}\left(u_{i+\frac{1}{2}, j+1}-u_{i+\frac{1}{2}, j}\right), \\
A_{y} u, D_{y} u \in \mathcal{V}_{x \times y} .
\end{array}
$$

The discrete Laplacian operator $\Delta_{h}: \mathcal{E}_{x \times \bar{y}}^{e w} \cup \mathcal{E}_{\bar{x} \times y}^{n s} \cup \mathcal{C}_{\bar{x} \times \bar{y}} \rightarrow \mathcal{E}_{x \times y}^{e w} \cup \mathcal{E}_{x \times y}^{n s} \cup \mathcal{C}_{x \times y}$ is defined as

$\Delta_{h} u=D_{x}\left(d_{x} u\right)+d_{y}\left(D_{y} u\right), \quad \Delta_{h} v=d_{x}\left(D_{x} v\right)+D_{y}\left(d_{y} v\right), \quad \Delta_{h} \phi=d_{x}\left(D_{x} \phi\right)+d_{y}\left(D_{y} \phi\right)$.

We discretize the physical variables that satisfy Neumann boundary conditions at the cell center and the ones that satisfy Dirichlet boundary conditions at the edge center. So, the cell-centered functions $\phi, \mu \in \mathcal{C}_{\bar{x} \times \bar{y}}$ satisfy homogeneous Neumann boundary conditions if and only if

$$
\begin{gathered}
\phi_{0, j}=\phi_{1, j}, \quad \phi_{N_{x}, j}=\phi_{N_{x}+1, j}, \quad \mu_{0, j}=\mu_{1, j}, \quad \begin{array}{r}
\mu_{N_{x}, j}=\mu_{N_{x}+1, j}, \\
j=1,2, \ldots, N_{y},
\end{array} \\
\phi_{i, 0}=\phi_{i, 1}, \quad \phi_{i, N_{y}}=\phi_{i, N_{y}+1}, \quad \mu_{i, 0}=\mu_{i, 1}, \quad \begin{array}{r}
\mu_{i, N_{y}}=\mu_{i, N_{y}+1}, \\
i=0,1, \ldots, N_{x}+1 .
\end{array}
\end{gathered}
$$

The velocity $\mathbf{v}=(u, v)$ (for $u \in \mathcal{E}_{x \times \bar{y}}^{e w}, v \in \mathcal{E}_{\bar{x} \times y}^{n s}$ ) satisfies the no-slip (Dirichlet) boundary conditions $\left.\mathbf{v}\right|_{\Omega}=0$ if and only if

$$
\begin{aligned}
u_{\frac{1}{2}, j}=u_{N_{x}+\frac{1}{2}, j}=0, & j=1,2, \ldots, N_{y}, \\
A_{y} u_{i+\frac{1}{2}, \frac{1}{2}}=A_{y} u_{i+\frac{1}{2}, N_{y}+\frac{1}{2}}=0, & i=0,1, \ldots, N_{x}, \\
v_{i, \frac{1}{2}}=v_{i, N_{y}+\frac{1}{2}} & =0, \quad i=1,2, \ldots, N_{x}, \\
A_{x} v_{\frac{1}{2}, j+\frac{1}{2}}=A_{x} v_{N_{x}+\frac{1}{2}, j+\frac{1}{2}} & =0, \quad j=0,1, \ldots, N_{y} .
\end{aligned}
$$

It is easy to show that

$$
D_{x} \phi, D_{x} \mu, u \in \mathcal{E}_{x \times y}^{e w 0}, \quad D_{y} \phi, D_{y} \mu, v \in \mathcal{E}_{x \times y}^{n s 0}, \quad A_{y} u, A_{x} v \in \mathcal{V}_{x \times y}^{0} .
$$

Based on the above definitions, we define the discrete 2D weighted inner products,

$$
\begin{aligned}
& (\phi, \psi)_{2}=h_{x} h_{y} \sum_{i=1}^{N_{x}} \sum_{j=1}^{N_{y}} \phi_{i, j} \psi_{i, j}, \\
& {[u, r]_{e w}=\left(a_{x}(u r), 1\right)_{2}, \quad[v, w]_{n s}=\left(a_{y}(v w), 1\right)_{2}, \quad\langle f, g\rangle_{v c}=\left(a_{x}\left(a_{y}(f g)\right), 1\right)_{2},}
\end{aligned}
$$

Copyright (c) by SIAM. Unauthorized reproduction of this article is prohibited. 
and the corresponding discrete norms,

$$
\|\phi\|_{2}=(\phi, \phi)_{2}^{\frac{1}{2}}, \quad\|u\|_{e w}=[u, u]_{e w}^{\frac{1}{2}}, \quad\|v\|_{n s}=[v, v]_{n s}^{\frac{1}{2}}, \quad\|f\|_{v c}=\langle f, f\rangle_{v c}^{\frac{1}{2}}
$$

For $\phi \in C_{\bar{x} \times \bar{y}}$, we define the following norm:

$$
\|\nabla \phi\|_{2}:=\sqrt{\left\|D_{x} \phi\right\|_{e w}^{2}+\left\|D_{y} \phi\right\|_{n s}^{2}} .
$$

For the edge-centered velocity $\mathbf{v}=(u, v), u \in \mathcal{E}_{x \times \bar{y}}^{e w}, v \in \mathcal{E}_{\bar{x} \times y}^{n s}$, we define the norms

$$
\|\mathbf{v}\|_{2}:=\sqrt{\|u\|_{e w}^{2}+\|v\|_{n s}^{2}}, \quad\|\nabla \mathbf{v}\|_{2}:=\sqrt{\left\|d_{x} u\right\|_{2}^{2}+\left\|D_{y} u\right\|_{v c}^{2}+\left\|D_{x} v\right\|_{v c}^{2}+\left\|d_{y} v\right\|_{2}^{2}} .
$$

Next, we introduce some useful lemmas. ties:

Lemma 2.1. For $\phi \in \mathcal{C}_{\bar{x} \times \bar{y}}, u \in \mathcal{E}_{x \times y}^{e w 0}, v \in \mathcal{E}_{x \times y}^{n s 0}$, there exist the following identi-

$$
\begin{aligned}
& {\left[A_{x} \phi, u\right]_{e w}=\left(\phi, a_{x} u\right)_{2}, \quad\left[D_{x} \phi, u\right]_{e w}+\left(\phi, d_{x} u\right)_{2}=0} \\
& {\left[A_{y} \phi, v\right]_{n s}=\left(\phi, a_{y} v\right)_{2}, \quad\left[D_{y} \phi, v\right]_{n s}+\left(\phi, d_{y} v\right)_{2}=0 .}
\end{aligned}
$$

Lemma 2.2. For $f \in \mathcal{V}_{x \times y}^{0}, u \in \mathcal{E}_{x \times \bar{y}}^{e w}, v \in \mathcal{E}_{\bar{x} \times y}^{n s}$, there exists the identities

$$
\left[a_{y} f, u\right]_{e w}=\left\langle f, A_{y} u\right\rangle_{v c}, \quad\left[a_{x} f, v\right]_{n s}=\left\langle f, A_{x} v\right\rangle_{v c} .
$$

Lemma 2.3. For $f \in \mathcal{V}_{x \times y}, u \in \mathcal{E}_{x \times \bar{y}}^{e w}, v \in \mathcal{E}_{\bar{x} \times y}^{n s}$, and $A_{y} u, A_{x} v \in \mathcal{V}_{x \times y}^{0}$, there exist the identities

$$
\begin{aligned}
& {\left[d_{y} f, u\right]_{e w}+\left\langle f, D_{y} u\right\rangle_{v c}=0} \\
& {\left[d_{x} f, v\right]_{n s}+\left\langle f, D_{x} v\right\rangle_{v c}=0 .}
\end{aligned}
$$

Throughout this paper, the results are proved for physical boundary conditions (1.2), but they are equally valid for periodic boundary conditions, or combinations of physical and periodic boundary conditions. We next discuss how to design efficient energy stable numerical schemes on staggered grids for the hydrodynamic phase field model subject to physical boundary conditions.

\section{Second order spatial discretization.}

3.1. Model reformulation and energy dissipation law. We first reformulate the governing system of equations to an equivalent form suitable for designing energy stable schemes. By introducing a new variable $q=\sqrt{f(\phi)}$ where we assume $f(\phi)>0$, system $(1.1)$ can be written as follows $[13,14,15]$ :

$$
\left\{\begin{array}{l}
\rho\left(u_{t}+\frac{1}{2}\left(u u_{x}+\left(u^{2}\right)_{x}\right)+\frac{1}{2}\left(v u_{y}+(u v)_{y}\right)\right)=-p_{x}+\eta \Delta u-\phi \mu_{x} \\
\rho\left(v_{t}+\frac{1}{2}\left(u v_{x}+(u v)_{x}\right)+\frac{1}{2}\left(v v_{y}+\left(v^{2}\right)_{y}\right)\right)=-p_{y}+\eta \Delta v-\phi \mu_{y} \\
u_{x}+v_{y}=0 \\
\phi_{t}+(\phi u)_{x}+(\phi v)_{y}=M \Delta \mu \\
\mu=2 q g(\phi)-\gamma_{1} \Delta \phi \\
q_{t}=g(\phi) \phi_{t}
\end{array}\right.
$$

Copyright $@$ ㅇ by SIAM. Unauthorized reproduction of this article is prohibited. 
where $g(\phi)=\frac{f^{\prime}(\phi)}{2 \sqrt{f(\phi)}}$. For the double-well free energy, we have $g(\phi)=\sqrt{\gamma_{2}}(1-2 \phi)$. For the Flory-Huggins free energy, we modify $f(\phi)$ as follows:

$$
f(\phi)=\gamma_{2}\left(\frac{\phi}{N_{1}} \ln \phi+\frac{(1-\phi)}{N_{2}} \ln (1-\phi)+\chi \phi(1-\phi)+C_{0}\right),
$$

where $C_{0}$ is taken as $\frac{1}{N_{1}}+\frac{1}{N_{2}}$. It is readily shown that $f(\phi)>0$. Notice that the additional constant $C_{0}$ in the potential does not affect dynamics of the system. Then

$$
g(\phi)=\frac{\gamma_{2}}{2 \sqrt{f(\phi)}}\left(\frac{\ln \phi}{N_{1}}-\frac{\ln (1-\phi)}{N_{2}}+\frac{1}{N_{1}}-\frac{1}{N_{2}}+\chi(1-2 \phi)\right) .
$$

THEOREM 3.1. With boundary conditions (1.2), the solution of system (3.1) satisfies the mass conservation law

$$
\frac{d}{d t}(\phi, 1)=0
$$

and the energy dissipation law

$$
\frac{d}{d t} E+\eta\|\nabla \mathbf{v}\|^{2}+M\|\nabla \mu\|^{2}=0,
$$

where the energy of system (3.1) is defined as

$$
E=\frac{\rho}{2}\|\mathbf{v}\|^{2}+\frac{\gamma_{1}}{2}\|\nabla \phi\|^{2}+\|q\|^{2} .
$$

3.2. Spatial discretization. Applying staggered-grid finite differences in space to system (3.1), we obtain a semidiscrete scheme as follows:

$$
\begin{aligned}
& \left\{\rho\left(\frac{d}{d t} u+\frac{1}{2}\left(u D_{x}\left(a_{x} u\right)+A_{x}\left(d_{x} u^{2}\right)\right)+\frac{1}{2}\left(a_{y}\left(A_{x} v D_{y} u\right)+d_{y}\left(A_{y} u A_{x} v\right)\right)\right)\right. \\
& \left.\quad=-D_{x} p+\eta \Delta_{h} u-A_{x} \phi D_{x} \mu\right\}\left.\right|_{i+\frac{1}{2}, j}, i=1, \ldots, N_{x}-1, j=1, \ldots, N_{y}, \\
& \left\{\rho\left(\frac{d}{d t} v+\frac{1}{2}\left(a_{x}\left(A_{y} u D_{x} v\right)+d_{x}\left(A_{y} u A_{x} v\right)\right)+\frac{1}{2}\left(v D_{y}\left(a_{y} v\right)+A_{y}\left(d_{y} v^{2}\right)\right)\right)\right. \\
& \left.\quad=-D_{y} p+\eta \Delta_{h} v-A_{y} \phi D_{y} \mu\right\}\left.\right|_{i, j+\frac{1}{2}}, i=1, \ldots, N_{x}, j=1, \ldots, N_{y}-1, \\
& \left.\left\{d_{x} u+d_{y} v=0\right\}\right|_{i, j}, i=1, \ldots, N_{x}, j=1, \ldots, N_{y}, \\
& \left.\left\{\frac{d}{d t} \phi+d_{x}\left(A_{x} \phi u\right)+d_{y}\left(A_{y} \phi v\right)=M \Delta_{h} \mu\right\}\right|_{i, j}, i=1, \ldots, N_{x}, j=1, \ldots, N_{y}, \\
& \left.\left\{\mu=2 q g(\phi)-\gamma_{1} \Delta_{h} \phi\right\}\right|_{i, j}, i=1, \ldots, N_{x}, j=1, \ldots, N_{y}, \\
& \left.\left\{\frac{d}{d t} q=g(\phi) \frac{d}{d t} \phi\right\}\right|_{i, j}, i=1, \ldots, N_{x}, j=1, \ldots, N_{y},
\end{aligned}
$$

where $u \in \mathcal{E}_{x \times \bar{y}}^{e w}, v \in \mathcal{E}_{\bar{x} \times y}^{n s}, \phi, \mu \in \mathcal{C}_{\bar{x} \times \bar{y}}$ satisfy boundary conditions (2.1)-(2.6), and $p, q \in \mathcal{C}_{x \times y}$.

THEOREM 3.2. The semidiscrete system given in (3.7) preserves the discrete mass conservation law given by

$$
\frac{d}{d t}(\phi, 1)_{2}=0
$$

Copyright (c) by SIAM. Unauthorized reproduction of this article is prohibited. 
and the discrete energy dissipation law given by

$$
\frac{d}{d t} E_{h}+\eta\|\nabla \mathbf{v}\|_{2}^{2}+M\|\nabla \mu\|_{2}^{2}=0
$$

where $E_{h}$ is the discrete energy functional defined as

$$
E_{h}=\frac{\rho}{2}\|\mathbf{v}\|_{2}^{2}+\frac{\gamma_{1}}{2}\|\nabla \phi\|_{2}^{2}+\|q\|_{2}^{2} .
$$

Proof. Computing the discrete inner product of (3.7d) with constant function 1, and using (2.7) and Lemma 2.1, we obtain (3.8).

Noticing that $u \in \mathcal{E}_{x \times y}^{e w 0}$ and using Lemma 2.1, we have

$$
\left[u D_{x}\left(a_{x} u\right)+A_{x}\left(d_{x} u^{2}\right), u\right]_{e w}=-\left(a_{x} u, d_{x} u^{2}\right)_{2}+\left(d_{x} u^{2}, a_{x} u\right)_{2}=0 .
$$

Noticing $A_{y} u, A_{x} v \in \mathcal{V}_{x \times y}^{0}$ and applying Lemmas 2.2 and 2.3, we have

$$
\left[a_{y}\left(A_{x} v D_{y} u\right)+d_{y}\left(A_{y} u A_{x} v\right), u\right]_{e w}=\left\langle A_{x} v D_{y} u, A_{y} u\right\rangle_{v c}-\left\langle A_{y} u A_{x} v, D_{y} u\right\rangle_{v c}=0 .
$$

Similarly, we can deduce

$$
\begin{aligned}
& {\left[a_{x}\left(A_{y} u D_{x} v\right)+d_{x}\left(A_{y} u A_{x} v\right), v\right]_{n s}=0, \quad\left[v D_{y}\left(a_{y} v\right)+A_{y}\left(d_{y} v^{2}\right), v\right]_{n s}=0} \\
& {\left[D_{x} p, u\right]_{e w}+\left[D_{y} p, v\right]_{n s}=-\left(p, d_{x} u+d_{y} v\right)_{2}=0,} \\
& {\left[A_{x} \phi D_{x} \mu, u\right]_{e w}+\left[A_{y} \phi D_{y} \mu, v\right]_{n s}=-\left(\mu, d_{x}\left(A_{x} \phi u\right)+d_{y}\left(A_{y} \phi v\right)\right)_{2},} \\
& {\left[\Delta_{h} u, u\right]_{e w}+\left[\Delta_{h} v, v\right]_{n s}=-\|\nabla \mathbf{v}\|_{2}^{2}, \quad\left(\Delta_{h} \phi, \phi\right)_{2}=-\|\nabla \phi\|_{2}^{2},} \\
& \quad\left(\Delta_{h} \mu, \mu\right)_{2}=-\|\nabla \mu\|_{2}^{2} .
\end{aligned}
$$

Computing the discrete inner product of (3.7a) and (3.7b) with $u$ and $v$, respectively, then adding the results and using (3.11)-(3.17), we have

$$
\rho\left(\left[u, u_{t}\right]_{e w}+\left[v, v_{t}\right]_{n s}\right)=-\eta\|\nabla \mathbf{v}\|_{2}^{2}+\left(\mu, d_{x}\left(A_{x} \phi u\right)+d_{y}\left(A_{y} \phi v\right)\right)_{2} .
$$

Similarly, we take the discrete inner product of (3.7d) with $\mu$ and obtain

$$
\left(\mu, \phi_{t}\right)_{2}=-\left(\mu, d_{x}\left(A_{x} \phi u\right)+d_{y}\left(A_{y} \phi v\right)\right)_{2}-M\|\nabla \mu\|_{2}^{2} .
$$

Adding (3.17) and (3.18) leads to

$$
\rho\left(\left[u, u_{t}\right]_{e w}+\left[v, v_{t}\right]_{n s}\right)+\left(\mu, \phi_{t}\right)_{2}=-\eta\|\nabla \mathbf{v}\|_{2}^{2}-M\|\nabla \mu\|_{2}^{2} .
$$

By a straightforward calculation, we have

$$
\begin{aligned}
\frac{d}{d t} E_{h} & =\rho\left(\left[u, u_{t}\right]_{e w}+\left[v, v_{t}\right]_{n s}\right)+\gamma_{1}\left(\left[D_{x} \phi, D_{x} \phi_{t}\right]_{e w}+\left[D_{y} \phi, D_{y} \phi_{t}\right]_{n s}\right)+\left(2 q, q_{t}\right)_{2} \\
& =\rho\left(\left[u, u_{t}\right]_{e w}+\left[v, v_{t}\right]_{n s}\right)-\gamma_{1}\left(\Delta_{h} \phi, \phi_{t}\right)_{2}+\left(2 q g(\phi), \phi_{t}\right)_{2} \\
& =\rho\left(\left[u, u_{t}\right]_{e w}+\left[v, v_{t}\right]_{n s}\right)+\left(\mu, \phi_{t}\right)_{2}=-\eta\|\nabla \mathbf{v}\|_{2}^{2}-M\|\nabla \mu\|_{2}^{2}
\end{aligned}
$$

which leads to (3.9).

Copyright $@$ by SIAM. Unauthorized reproduction of this article is prohibited. 


\section{Fully discrete schemes and their unconditional stability.}

4.1. Second order fully discrete linear scheme. Applying the linear-implicit Crank-Nicolson method in time to system (3.7), we obtain a fully discrete numerical scheme:

$$
\begin{gathered}
\rho\left[\delta_{t}^{+} u^{n}+\frac{1}{2}\left(\bar{u}^{n+\frac{1}{2}} D_{x}\left(a_{x} u^{n+\frac{1}{2}}\right)+A_{x}\left(d_{x}\left(u^{n+\frac{1}{2}} \bar{u}^{n+\frac{1}{2}}\right)\right)+a_{y}\left(A_{x} \bar{v}^{n+\frac{1}{2}} D_{y} u^{n+\frac{1}{2}}\right)\right.\right. \\
\left.\left.+d_{y}\left(A_{y} u^{n+\frac{1}{2}} A_{x} \bar{v}^{n+\frac{1}{2}}\right)\right)\right]=-D_{x} p^{n+\frac{1}{2}}+\eta \Delta_{h} u^{n+\frac{1}{2}}-A_{x} \bar{\phi}^{n+\frac{1}{2}} D_{x} \mu^{n+\frac{1}{2}}
\end{gathered}
$$

$$
\begin{gathered}
\rho\left[\delta_{t}^{+} v^{n}+\frac{1}{2}\left(a_{x}\left(A_{y} \bar{u}^{n+\frac{1}{2}} D_{x} v^{n+\frac{1}{2}}\right)+d_{x}\left(A_{y} \bar{u}^{n+\frac{1}{2}} A_{x} v^{n+\frac{1}{2}}\right)+\bar{v}^{n+\frac{1}{2}} D_{y}\left(a_{y} v^{n+\frac{1}{2}}\right)\right.\right. \\
\left.\left.+A_{y}\left(d_{y}\left(v^{n+\frac{1}{2}} \bar{v}^{n+\frac{1}{2}}\right)\right)\right)\right]=-D_{y} p^{n+\frac{1}{2}}+\eta \Delta_{h} v^{n+\frac{1}{2}}-A_{y} \bar{\phi}^{n+\frac{1}{2}} D_{y} \mu^{n+\frac{1}{2}}
\end{gathered}
$$

(4.1c) $d_{x} u^{n+\frac{1}{2}}+d_{y} v^{n+\frac{1}{2}}=0$,

$$
\begin{aligned}
& \delta_{t}^{+} \phi^{n}+d_{x}\left(A_{x} \bar{\phi}^{n+\frac{1}{2}} u^{n+\frac{1}{2}}\right)+d_{y}\left(A_{y} \bar{\phi}^{n+\frac{1}{2}} v^{n+\frac{1}{2}}\right)=M \Delta_{h} \mu^{n+\frac{1}{2}}, \\
& \text { (4.1e) } \mu^{n+\frac{1}{2}}=2 q^{n+\frac{1}{2}} \overline{g(\phi)}^{n+\frac{1}{2}}-\gamma_{1} \Delta_{h} \phi^{n+\frac{1}{2}} \\
& \text { (4.1f) } \delta_{t}^{+} q^{n}=\overline{g(\phi)}^{n+\frac{1}{2}} \delta_{t}^{+} \phi^{n}
\end{aligned}
$$

where $n \geq 0, u^{n+1} \in \mathcal{E}_{x \times \bar{y}}^{e w}, v^{n+1} \in \mathcal{E}_{\bar{x} \times y}^{n s}, \phi^{n+1}, \mu^{n+\frac{1}{2}} \in \mathcal{C}_{\bar{x} \times \bar{y}}$ satisfy boundary conditions (2.1)-(2.6), $p^{n+\frac{1}{2}}, q^{n+1} \in \mathcal{C}_{x \times y}$ and $\delta_{t}^{+} u^{n}=\left(u^{n+1}-u^{n}\right) / \Delta t, u^{n+\frac{1}{2}}=$ $\left(u^{n+1}+u^{n}\right) / 2, \bar{u}^{n+\frac{1}{2}}=\left(3 u^{n}-u^{n-1}\right) / 2$, etc. We define $u^{-1} \equiv u^{0}, v^{-1} \equiv v^{0}, \phi^{-1} \equiv \phi^{0}$. The spatial indices of system (4.1) are identical to those of system (3.7) and are thus omitted for simplicity. Note that we enforce the solvability condition

$$
\left(p^{n+\frac{1}{2}}, 1\right)_{2}=0
$$

in the scheme to eliminate the indeterminacy in the pressure field.

THEOREM 4.1. The linear scheme given in (4.1) preserves the discrete mass conservation law

$$
\left(\phi^{n+1}, 1\right)_{2}=\left(\phi^{n}, 1\right)_{2}
$$

and the discrete energy dissipation law

$$
\delta_{t}^{+} E_{h}^{n}+\eta\left\|\nabla \mathbf{v}^{n+\frac{1}{2}}\right\|_{2}^{2}+M\left\|\nabla \mu^{n+\frac{1}{2}}\right\|_{2}^{2}=0,
$$

where the discrete energy is defined as

$$
E_{h}^{n}=\frac{\rho}{2}\left\|\mathbf{v}^{n}\right\|_{2}^{2}+\frac{\gamma_{1}}{2}\left\|\nabla \phi^{n}\right\|_{2}^{2}+\left\|q^{n}\right\|_{2}^{2} .
$$

Proof. Assuming that $u^{0}, v^{0}, \phi^{0}$ satisfy discrete boundary conditions (2.1)-(2.6), we deduce inductively from system (4.1) that both $u^{n+\frac{1}{2}}, v^{n+\frac{1}{2}}, \phi^{n+\frac{1}{2}}, \mu^{n+\frac{1}{2}}$ and $\bar{u}^{n+\frac{1}{2}}$, $\bar{v}^{n+\frac{1}{2}}$ for $\forall n \in \mathbb{N}$ also satisfy the boundary conditions.

Analogous to the proof of Theorem 3.2, we take the discrete inner product of (4.1d) with constant function 1 and obtain

$$
\left(\delta_{t}^{+} \phi^{n}, 1\right)_{2}=0,
$$

Copyright $@$ by SIAM. Unauthorized reproduction of this article is prohibited. 
which implies (4.3). Furthermore, we can also deduce from (4.1) that $(4.7)$ $\rho\left(\left[u^{n+\frac{1}{2}}, \delta_{t}^{+} u^{n}\right]_{e w}+\left[v^{n+\frac{1}{2}}, \delta_{t}^{+} v^{n}\right]_{n s}\right)+\left(\mu^{n+\frac{1}{2}}, \delta_{t}^{+} \phi^{n}\right)_{2}=-\eta\left\|\nabla \mathbf{v}^{n+\frac{1}{2}}\right\|_{2}^{2}-M\left\|\nabla \mu^{n+\frac{1}{2}}\right\|_{2}^{2}$.

Using (4.7) and the identity $\delta_{t}^{+}\left(u^{n} \cdot v^{n}\right)=\delta_{t}^{+} u^{n} \cdot v^{n+\frac{1}{2}}+u^{n+\frac{1}{2}} \cdot \delta_{t}^{+} v^{n}$, we obtain

$$
\begin{aligned}
\delta_{t}^{+} E_{h}^{n}= & \rho\left(\left[u^{n+\frac{1}{2}}, \delta_{t}^{+} u^{n}\right]_{e w}+\left[v^{n+\frac{1}{2}}, \delta_{t}^{+} v^{n}\right]_{n s}\right) \\
& +\gamma_{1}\left(\left[D_{x} \phi^{n+\frac{1}{2}}, D_{x} \delta_{t}^{+} \phi^{n}\right]_{e w}+\left[D_{y} \phi^{n+\frac{1}{2}}, D_{y} \delta_{t}^{+} \phi^{n}\right]_{n s}\right)+\left(2 q^{n+\frac{1}{2}}, \delta_{t}^{+} q^{n}\right)_{2} \\
= & \rho\left(\left[u^{n+\frac{1}{2}}, \delta_{t}^{+} u^{n}\right]_{e w}+\left[v^{n+\frac{1}{2}}, \delta_{t}^{+} v^{n}\right]_{n s}\right)-\gamma_{1}\left(\Delta_{h} \phi^{n+\frac{1}{2}}, \delta_{t}^{+} \phi^{n}\right)_{2} \\
& +\left(2 q^{n+\frac{1}{2}} \overline{g(\phi)} \overline{n+\frac{1}{2}}^{n}, \delta_{t}^{+} \phi^{n}\right)_{2} \\
= & \rho\left(\left[u^{n+\frac{1}{2}}, \delta_{t}^{+} u^{n}\right]_{e w}+\left[v^{n+\frac{1}{2}}, \delta_{t}^{+} v^{n}\right]_{n s}\right)+\left(\mu^{n+\frac{1}{2}}, \delta_{t}^{+} \phi^{n}\right)_{2} \\
= & -\eta\left\|\nabla \mathbf{v}^{n+\frac{1}{2}}\right\|_{2}^{2}-M\left\|\nabla \mu^{n+\frac{1}{2}}\right\|_{2}^{2} .
\end{aligned}
$$

This completes the proof.

REMARK 4.1. If we replace all $\overline{(\cdot)}^{n+\frac{1}{2}}$ with $(\cdot)^{n+\frac{1}{2}}$ in (4.1), we obtain a second order nonlinear energy stable scheme. If we replace all $\overline{(\cdot)}^{n+\frac{1}{2}}$ with $(\cdot)^{n}$ in $(4.1)$, we obtain a two-level energy stable scheme, which is still linear but is of order 1 in time. In the numerical experiments, we use the two-level scheme to compute the initial data for the second level values of the three-level scheme given in (4.1). This does not affect the overall accuracy of second order scheme (4.1).

4.2. Linear decoupled scheme. The linear scheme given above is fully coupled. We next develop a decoupled linear scheme by introducing a "stabilizing term" in the velocity and solving the momentum balance equation using the projection method in two steps. We then show that the scheme is unconditionally energy stable and uniquely solvable. This new decoupled, linear scheme differs from the one developed by Chen and Shen in [7] in that we treat the convective term more precisely to warrant the energy stability.

The decoupled, fully discrete scheme is given as follows:

Step 1.

$$
\left\{\begin{array}{l}
\delta_{t}^{+} \phi^{n}+d_{x}\left(A_{x} \phi^{n} u_{*}^{n}\right)+d_{y}\left(A_{y} \phi^{n} v_{*}^{n}\right)=M \Delta_{h} \mu^{n+1} \\
\mu^{n+1}=2 q^{n+1} g\left(\phi^{n}\right)-\gamma_{1} \Delta_{h} \phi^{n+1} \\
\delta_{t}^{+} q^{n}=g\left(\phi^{n}\right) \delta_{t}^{+} \phi^{n}
\end{array}\right.
$$

where

$$
\left\{\begin{array}{l}
u_{*}^{n}=u^{n}-\frac{\Delta t}{\rho} A_{x} \phi^{n} D_{x} \mu^{n+1}, \\
v_{*}^{n}=v^{n}-\frac{\Delta t}{\rho} A_{y} \phi^{n} D_{y} \mu^{n+1},
\end{array}\right.
$$

and $\phi^{n+1}, \mu^{n+1} \in \mathcal{C}_{\bar{x} \times \bar{y}}$ satisfy discrete boundary conditions $(2.1)-(2.2), q^{n+1} \in \mathcal{C}_{x \times y}$.

$$
\text { Step } 2 \text {. }
$$

$$
\left\{\begin{array}{l}
\frac{\rho}{\Delta t}\left(\widetilde{u}^{n+1}-u^{n}\right)+\frac{\rho}{2}\left(u^{n} D_{x}\left(a_{x} \widetilde{u}^{n+1}\right)+A_{x}\left(d_{x}\left(\widetilde{u}^{n+1} u^{n}\right)\right)\right) \\
+\frac{\rho}{2}\left(a_{y}\left(A_{x} v^{n} D_{y} \widetilde{u}^{n+1}\right)+d_{y}\left(A_{y} \widetilde{u}^{n+1} A_{x} v^{n}\right)\right)=-D_{x} p^{n}+\eta \Delta_{h} \widetilde{u}^{n+1}-A_{x} \phi^{n} D_{x} \mu^{n+1}, \\
\frac{\rho}{\Delta t}\left(\widetilde{v}^{n+1}-v^{n}\right)+\frac{\rho}{2}\left(a_{x}\left(A_{y} u^{n} D_{x} \widetilde{v}^{n+1}\right)+d_{x}\left(A_{y} u^{n} A_{x} \widetilde{v}^{n+1}\right)\right) \\
+\frac{\rho}{2}\left(v^{n} D_{y}\left(a_{y} \widetilde{v}^{n+1}\right)+A_{y}\left(d_{y}\left(\widetilde{v}^{n+1} v^{n}\right)\right)\right)=-D_{y} p^{n}+\eta \Delta_{h} \widetilde{v}^{n+1}-A_{y} \phi^{n} D_{y} \mu^{n+1},
\end{array}\right.
$$

Copyright (c) by SIAM. Unauthorized reproduction of this article is prohibited. 
where $\widetilde{u}^{n+1} \in \mathcal{E}_{x \times \bar{y}}^{e w}, \widetilde{v}^{n+1} \in \mathcal{E}_{\bar{x} \times y}^{n s}$ satisfy discrete boundary conditions (2.3)-(2.6).

$$
\left\{\begin{array}{l}
\left.\left\{\frac{\rho}{\Delta t}\left(u^{n+1}-\widetilde{u}^{n+1}\right)+D_{x}\left(p^{n+1}-p^{n}\right)=0\right\}\right|_{i+\frac{1}{2}, j}, i=0, \ldots, N_{x}, j=0, \ldots, N_{y}+1, \\
\left.\left\{\frac{\rho}{\Delta t}\left(v^{n+1}-\widetilde{v}^{n+1}\right)+D_{y}\left(p^{n+1}-p^{n}\right)=0\right\}\right|_{i, j+\frac{1}{2}}, i=0, \ldots, N_{x}+1, j=0, \ldots, N_{y}, \\
\left.\left\{d_{x} u^{n+1}+d_{y} v^{n+1}=0\right\}\right|_{i, j}, i=1, \ldots, N_{x}, j=1, \ldots, N_{y}, \\
\left(p^{n+1}-p^{n}\right)_{0, j}=\left(p^{n+1}-p^{n}\right)_{1, j}, \quad\left(p^{n+1}-p^{n}\right)_{N_{x}, j}=\left(p^{n+1}-p^{n}\right)_{N_{x}+1, j}, \\
j=1,2, \ldots, N_{y}, \\
\left(p^{n+1}-p^{n}\right)_{i, 0}=\left(p^{n+1}-p^{n}\right)_{i, 1}, \quad\left(p^{n+1}-p^{n}\right)_{i, N_{y}}=\left(p^{n+1}-p^{n}\right)_{i, N_{y}+1}, \\
i=0,1, \ldots, N_{x}+1,
\end{array}\right.
$$

Step 3 .

where $u^{n+1} \in \mathcal{E}_{x \times \bar{y}}^{e w}, v^{n+1} \in \mathcal{E}_{\bar{x} \times y}^{n s}$, and $p^{n+1} \in \mathcal{C}_{\bar{x} \times \bar{y}}$ satisfies the solvability condition $\left(p^{n+1}, 1\right)_{2}=0$. The spatial indices of system (4.8) and system (4.10) are identical to those of system (3.7) and are thus omitted.

REMARK 4.2. The last step leads to

$$
\Delta_{h}\left(p^{n+1}-p^{n}\right)=\frac{\rho}{\Delta t}\left(d_{x} \widetilde{u}^{n+1}+d_{y} \widetilde{v}^{n+1}\right) .
$$

So we can first compute $p^{n+1}$ and then

$$
u^{n+1}=\widetilde{u}^{n+1}-\frac{\Delta t}{\rho} D_{x}\left(p^{n+1}-p^{n}\right), \quad v^{n+1}=\widetilde{v}^{n+1}-\frac{\Delta t}{\rho} D_{y}\left(p^{n+1}-p^{n}\right) .
$$

REMARK 4.3. In the scheme, computations of $\left(\phi^{n+1}, \mu^{n+1}, q^{n+1}\right), \widetilde{u}^{n+1}, \widetilde{v}^{n+1}$, $u^{n+1}, v^{n+1}$, and $p^{n+1}$ are totally decoupled!

THEOREM 4.2. The scheme given in (4.8)-(4.11) satisfies the discrete mass conservation law

$$
\left(\phi^{n+1}, 1\right)_{2}=\left(\phi^{n}, 1\right)_{2}
$$

and the discrete energy law

$$
\frac{1}{\Delta t}\left(E_{h}^{n+1}-E_{h}^{n}+\widetilde{E}_{h}^{n}\right)+\eta\left\|\nabla \widetilde{\mathbf{v}}^{n+1}\right\|_{2}^{2}+M\left\|\nabla \mu^{n+1}\right\|_{2}^{2}=0,
$$

where

$$
\begin{aligned}
& E_{h}^{n}=\frac{\rho}{2}\left\|\mathbf{v}^{n}\right\|_{2}^{2}+\frac{\gamma_{1}}{2}\left\|\nabla \phi^{n}\right\|_{2}^{2}+\left\|q^{n}\right\|_{2}^{2}+\frac{\Delta t^{2}}{2 \rho}\left\|\nabla p^{n}\right\|_{2}^{2}, \\
& \widetilde{E}^{n}=\frac{\rho}{2}\left(\left\|\widetilde{\mathbf{v}}^{n+1}-\mathbf{v}_{*}^{n}\right\|_{2}^{2}+\left\|\mathbf{v}_{*}^{n}-\mathbf{v}^{n}\right\|_{2}^{2}\right)+\frac{\gamma_{1}}{2}\left\|\nabla\left(\phi^{n+1}-\phi^{n}\right)\right\|_{2}^{2}+\left\|q^{n+1}-q^{n}\right\|_{2}^{2} .
\end{aligned}
$$

The decoupled scheme is therefore unconditionally energy stable.

Proof. According to the last two equations of (4.11), we have

$$
\begin{aligned}
& D_{x}\left(p^{n+1}-p^{n}\right)_{\frac{1}{2}, j}=D_{x}\left(p^{n+1}-p^{n}\right)_{N_{x}+\frac{1}{2}, j}=0, j=0, \ldots, N_{y}+1, \\
& D_{y}\left(p^{n+1}-p^{n}\right)_{i, \frac{1}{2}}=D_{y}\left(p^{n+1}-p^{n}\right)_{i, N_{y}+\frac{1}{2}}=0, i=0, \ldots, N_{x}+1,
\end{aligned}
$$

Copyright (c) by SIAM. Unauthorized reproduction of this article is prohibited. 
which lead to

$$
\begin{aligned}
& u_{\frac{1}{2}, j}^{n+1}=\widetilde{u}_{\frac{1}{2}, j}^{n+1}, u_{N_{x}+\frac{1}{2}, j}^{n+1}=\widetilde{u}_{N_{x}+\frac{1}{2}, j}^{n+1}, j=0, \ldots, N_{y}+1, \\
& v_{i, \frac{1}{2}}^{n+1}=\widetilde{v}_{i, \frac{1}{2}}^{n+1}, v_{i, N_{y}+\frac{1}{2}}^{n+1}=\widetilde{v}_{i, N_{y}+\frac{1}{2}}^{n+1}, i=0, \ldots, N_{x}+1 .
\end{aligned}
$$

Since $\widetilde{u}^{n+1}, \widetilde{v}^{n+1}$ satisfy the boundary conditions (2.3)-(2.6), we obtain

$$
\begin{aligned}
& \widetilde{u}_{\frac{1}{2}, j}^{n+1}=\widetilde{u}_{N_{x}+\frac{1}{2}, j}^{n+1}=0, j=0, \ldots, N_{y}+1, \\
& \widetilde{v}_{i, \frac{1}{2}}^{n+1}=\widetilde{v}_{i, N_{y}+\frac{1}{2}}^{n+1}=0, i=0, \ldots, N_{x}+1,
\end{aligned}
$$

and

$$
\widetilde{u}^{n+1} \in \mathcal{E}_{x \times y}^{e w 0}, \quad \widetilde{v}^{n+1} \in \mathcal{E}_{x \times y}^{n s 0}, \quad A_{y} \widetilde{u}^{n+1}, A_{x} \widetilde{v}^{n+1} \in \mathcal{V}_{x \times y}^{0} .
$$

Combining (4.15), (4.16), (4.17), and (4.18) leads to

$$
\begin{gathered}
u_{\frac{1}{2}, j}^{n+1}=u_{N_{x}+\frac{1}{2}, j}^{n+1}=0, j=0, \ldots, N_{y}+1, \\
v_{i, \frac{1}{2}}^{n+1}=v_{i, N_{y}+\frac{1}{2}}^{n+1}=0, i=0, \ldots, N_{x}+1 .
\end{gathered}
$$

Assume that $u^{0}, v^{0}$ satisfy the boundary conditions (4.20) and (4.21). Then $u^{n}, v^{n} \forall n \in$ $\mathbb{N}$ satisfy the boundary conditions (4.20) and (4.21), which imply

$$
\begin{aligned}
& A_{y} u_{\frac{1}{2}, j+\frac{1}{2}}^{n}=A_{y} u_{N_{x}+\frac{1}{2}, j+\frac{1}{2}}^{n}=0, j=0, \ldots, N_{y}, \\
& A_{x} v_{i+\frac{1}{2}, \frac{1}{2}}^{n}=A_{x} v_{i+\frac{1}{2}, N_{y}+\frac{1}{2}}^{n}=0, i=0, \ldots, N_{x} .
\end{aligned}
$$

In addition, we can deduce from (4.17) and (4.18) that

$$
\begin{aligned}
& D_{y} \widetilde{u}_{\frac{1}{2}, j+\frac{1}{2}}^{n+1}=D_{y} \widetilde{u}_{N_{x}+\frac{1}{2}, j+\frac{1}{2}}^{n+1}=0, j=0, \ldots, N_{y}, \\
& D_{x} \widetilde{v}_{i+\frac{1}{2}, \frac{1}{2}}^{n+1}=D_{x} \widetilde{v}_{i+\frac{1}{2}, N_{y}+\frac{1}{2}}^{n+1}=0, i=0, \ldots, N_{x} .
\end{aligned}
$$

Therefore, we have

$$
u^{n+1} \in \mathcal{E}_{x \times y}^{e w 0}, \quad v^{n+1} \in \mathcal{E}_{x \times y}^{n s 0}, \quad A_{x} v^{n} D_{y} \widetilde{u}^{n+1}, A_{y} u^{n} D_{x} \widetilde{v}^{n+1} \in \mathcal{V}_{x \times y}^{0} .
$$

Similarly, we obtain

$$
D_{x} \phi^{n+1}, D_{x} \mu^{n+1}, u_{*}^{n} \in \mathcal{E}_{x \times y}^{e w 0}, \quad D_{y} \phi^{n+1}, D_{y} \mu^{n+1}, v_{*}^{n} \in \mathcal{E}_{x \times y}^{n s 0} .
$$

Note that conditions (4.19), (4.26), and (4.27) are important for using the corresponding discrete summation-by-parts formulas proposed in Lemmas 2.1-2.3.

Due to condition (4.27), we compute the discrete inner product of the first line of (4.8) with constant function 1 and obtain (4.13).

Taking the discrete inner product of the two equations in (4.10) with $2 \widetilde{u}^{n+1}$ and $2 \widetilde{v}^{n+1}$, respectively, then adding the results and using (4.9), Lemmas 2.1-2.3, and the equality $2 a(a-b)=a^{2}-b^{2}+(a-b)^{2}$, we obtain

$$
\begin{aligned}
& \frac{\rho}{\Delta t}\left(\left\|\widetilde{\mathbf{v}}^{n+1}\right\|_{2}^{2}-\left\|\mathbf{v}_{*}^{n}\right\|_{2}^{2}+\left\|\widetilde{\mathbf{v}}^{n+1}-\mathbf{v}_{*}^{n}\right\|_{2}^{2}\right) \\
= & -2\left(\left[\widetilde{u}^{n+1}, D_{x} p^{n}\right]_{e w}+\left[\widetilde{v}^{n+1}, D_{y} p^{n}\right]_{n s}\right)-2 \eta\left\|\nabla \widetilde{\mathbf{v}}^{n+1}\right\|_{2}^{2} .
\end{aligned}
$$

Copyright $@$ by SIAM. Unauthorized reproduction of this article is prohibited. 
To deal with the first term on the right-hand side in the above equality, taking the discrete inner product of the first equation of (4.11) with $\frac{2 \Delta t}{\rho} D_{x} p^{n}$ and that of the second equation of (4.11) with $\frac{2 \Delta t}{\rho} D_{y} p^{n}$, respectively, then adding the results and using (2.8), (2.9), and the equality $d_{x} u^{n+1}+d_{y} v^{n+1}=0$, we obtain

$2\left(\left[\widetilde{u}^{n+1}, D_{x} p^{n}\right]_{e w}+\left[\widetilde{v}^{n+1}, D_{y} p^{n}\right]_{n s}\right)=\frac{\Delta t}{\rho}\left(\left\|\nabla p^{n+1}\right\|_{2}^{2}-\left\|\nabla p^{n}\right\|_{2}^{2}-\left\|\nabla\left(p^{n+1}-p^{n}\right)\right\|_{2}^{2}\right) ;$

we also derive from (4.11) that

$$
\left\|\nabla\left(p^{n+1}-p^{n}\right)\right\|_{2}^{2}=\frac{\rho^{2}}{\Delta t^{2}}\left\|\mathbf{v}^{n+1}-\widetilde{\mathbf{v}}^{n+1}\right\|_{2}^{2}
$$

we then take the discrete inner product of the first equation of (4.11) with $2 u^{n+1}$ and that of the second equation of (4.11) with $2 v^{n+1}$, respectively, and add the results:

$$
\left\|\mathbf{v}^{n+1}-\widetilde{\mathbf{v}}^{n+1}\right\|_{2}^{2}=\left\|\widetilde{\mathbf{v}}^{n+1}\right\|_{2}^{2}-\left\|\mathbf{v}^{n+1}\right\|_{2}^{2} .
$$

Combining the above four equalities, we have

$\frac{\rho}{\Delta t}\left(\left\|\mathbf{v}^{n+1}\right\|_{2}^{2}-\left\|\mathbf{v}_{*}^{n}\right\|_{2}^{2}+\left\|\widetilde{\mathbf{v}}^{n+1}-\mathbf{v}_{*}^{n}\right\|_{2}^{2}\right)+\frac{\Delta t}{\rho}\left(\left\|\nabla p^{n+1}\right\|_{2}^{2}-\left\|\nabla p^{n}\right\|_{2}^{2}\right)=-2 \eta\left\|\nabla \widetilde{\mathbf{v}}^{n+1}\right\|_{2}^{2}$.

Taking the discrete inner product of the first equation of (4.9) with $2 u_{*}^{n}$ and of the second equation of (4.9) with $2 v_{*}^{n}$, respectively, then adding the results, we obtain $(4.33)$

$\frac{\rho}{\Delta t}\left(\left\|\mathbf{v}_{*}^{n}\right\|_{2}^{2}-\left\|\mathbf{v}^{n}\right\|_{2}^{2}+\left\|\mathbf{v}_{*}^{n}-\mathbf{v}^{n}\right\|_{2}^{2}\right)=-2\left(\left[A_{x} \phi^{n} u_{*}^{n}, D_{x} \mu^{n+1}\right]_{e w}+\left[A_{y} \phi^{n} v_{*}^{n}, D_{y} \mu^{n+1}\right]_{n s}\right)$.

Adding (4.33) to (4.32), we have

$$
\begin{aligned}
& \frac{\rho}{\Delta t}\left(\left\|\mathbf{v}^{n+1}\right\|_{2}^{2}-\left\|\mathbf{v}^{n}\right\|_{2}^{2}+\left\|\widetilde{\mathbf{v}}^{n+1}-\mathbf{v}_{*}^{n}\right\|_{2}^{2}+\left\|\mathbf{v}_{*}^{n}-\mathbf{v}^{n}\right\|_{2}^{2}\right)+\frac{\Delta t}{\rho}\left(\left\|\nabla p^{n+1}\right\|_{2}^{2}-\left\|\nabla p^{n}\right\|_{2}^{2}\right) \\
= & -2 \eta\left\|\nabla \widetilde{\mathbf{v}}^{n+1}\right\|_{2}^{2}-2\left(\left[A_{x} \phi^{n} u_{*}^{n}, D_{x} \mu^{n+1}\right]_{e w}+\left[A_{y} \phi^{n} v_{*}^{n}, D_{y} \mu^{n+1}\right]_{n s}\right) .
\end{aligned}
$$

Taking the discrete inner product of the first equation of (4.8) with $2 \mu^{n+1}$, we have $(4.35)$

$\frac{2}{\Delta t}\left(\phi^{n+1}-\phi^{n}, \mu^{n+1}\right)_{2}=2\left(\left[A_{x} \phi^{n} u_{*}^{n}, D_{x} \mu^{n+1}\right]_{e w}+\left[A_{y} \phi^{n} v_{*}^{n}, D_{y} \mu^{n+1}\right]_{n s}\right)-2 M\left\|\nabla \mu^{n+1}\right\|_{2}^{2}$.

Taking the discrete inner product of the second equation of (4.8) with $-\frac{2}{\Delta t}\left(\phi^{n+1}-\phi^{n}\right)$, we obtain

$$
\begin{aligned}
-\frac{2}{\Delta t}\left(\phi^{n+1}-\phi^{n}, \mu^{n+1}\right)_{2}= & -\frac{4}{\Delta t}\left(\phi^{n+1}-\phi^{n}, q^{n+1} g\left(\phi^{n}\right)\right)_{2} \\
& -\frac{\gamma_{1}}{\Delta t}\left(\left\|\nabla \phi^{n+1}\right\|_{2}^{2}-\left\|\nabla \phi^{n}\right\|_{2}^{2}+\left\|\nabla\left(\phi^{n+1}-\phi^{n}\right)\right\|_{2}^{2}\right) .
\end{aligned}
$$

Taking the discrete inner product of the third equation of (4.8) with $4 q^{n+1}$, we have

$$
\frac{2}{\Delta t}\left(\left\|q^{n+1}\right\|_{2}^{2}-\left\|q^{n}\right\|_{2}^{2}+\left\|q^{n+1}-q^{n}\right\|_{2}^{2}\right)=\frac{4}{\Delta t}\left(\phi^{n+1}-\phi^{n}, q^{n+1} g\left(\phi^{n}\right)\right)_{2} .
$$

Adding (4.34), (4.35), (4.36), and (4.37) leads to (4.14).

Copyright $($ C by SIAM. Unauthorized reproduction of this article is prohibited. 
REMARK 4.4. We deduce from (4.14) that the inclusion of the excessive energy dissipation term $\tilde{E}^{n}$ enhances the energy dissipation, making the scheme "more" dissipative in order to achieve decoupling of the equations. It then follows that

$$
E_{h}^{n+1} \leq E_{h}^{n}
$$

which implies that the fully discrete scheme (4.8)-(4.11) is unconditionally stable.

For the linear systems given by the linear schemes, their unique solvability is an issue that must be addressed. Next, we show that the linear systems are indeed solvable uniquely.

\section{Unique solvability of the linear systems.}

5.1. Unique solvability of the second order coupled scheme. For brevity, we denote $u:=u^{n+\frac{1}{2}}, \bar{u}:=\bar{u}^{n+\frac{1}{2}}$, etc. Then the system (4.1) can be written as

$$
\begin{aligned}
& \rho\left(\frac{2}{\Delta t}\left(u-u^{n}\right)+\frac{1}{2}\left(\bar{u} D_{x}\left(a_{x} u\right)+A_{x}\left(d_{x}(u \bar{u})\right)\right)+\frac{1}{2}\left(a_{y}\left(A_{x} \bar{v} D_{y} u\right)+d_{y}\left(A_{y} u A_{x} \bar{v}\right)\right)\right) \\
& =-D_{x} p+\eta \Delta_{h} u-A_{x} \bar{\phi} D_{x} \mu,
\end{aligned}
$$

$$
\begin{aligned}
& \rho\left(\frac{2}{\Delta t}\left(v-v^{n}\right)+\frac{1}{2}\left(a_{x}\left(A_{y} \bar{u} D_{x} v\right)+d_{x}\left(A_{y} \bar{u} A_{x} v\right)\right)+\frac{1}{2}\left(\bar{v} D_{y}\left(a_{y} v\right)+A_{y}\left(d_{y}(v \bar{v})\right)\right)\right) \\
& =-D_{y} p+\eta \Delta_{h} v-A_{y} \bar{\phi} D_{y} \mu,
\end{aligned}
$$

$$
\begin{aligned}
& d_{x} u+d_{y} v=0, \\
& \frac{2}{\Delta t}\left(\phi-\phi^{n}\right)+d_{x}\left(A_{x} \bar{\phi} u\right)+d_{y}\left(A_{y} \bar{\phi} v\right)=M \Delta_{h} \mu, \\
& \mu=2 q \overline{g(\phi)}-\gamma_{1} \Delta_{h} \phi, \\
& q=\overline{g(\phi)} \phi+q^{n}-\overline{g(\phi)} \phi^{n},
\end{aligned}
$$

where $u \in \mathcal{E}_{x \times \bar{y}}^{e w}, v \in \mathcal{E}_{\bar{x} \times y}^{n s}, \phi, \mu \in \mathcal{C}_{\bar{x} \times \bar{y}}$ satisfy boundary conditions (2.1)-(2.6), and $p, q \in \mathcal{C}_{x \times y}$. Note that the number of equations in the linear system (5.1) is equal to the number of unknowns. However, noticing $u \in \mathcal{E}_{x \times y}^{e w 0}, v \in \mathcal{E}_{x \times y}^{n s 0}$, it is readily proven that $\left(d_{x} u+d_{y} v, 1\right)_{2}=0$, which implies that the linear system is singular due to (5.1c). To make it uniquely solvable, we replace one equation of (5.1c) with the solvability condition $(p, 1)_{2}=0$. Next, we have the following theorem.

Theorem 5.1. For any $\rho, \eta, \gamma_{1}, M, \Delta t>0$, linear system (5.1) with the solvability condition $(p, 1)_{2}=0$ is uniquely solvable. Therefore, the linear scheme (4.1) with (4.2) is uniquely solvable.

Proof. We first consider the following homogeneous linear equation system:

$$
\begin{aligned}
& \rho\left(\frac{2}{\Delta t} u+\frac{1}{2}\left(\bar{u} D_{x}\left(a_{x} u\right)+A_{x}\left(d_{x}(u \bar{u})\right)\right)+\frac{1}{2}\left(a_{y}\left(A_{x} \bar{v} D_{y} u\right)+d_{y}\left(A_{y} u A_{x} \bar{v}\right)\right)\right) \\
& =-D_{x} p+\eta \Delta_{h} u-A_{x} \bar{\phi} D_{x} \mu, \\
& \rho\left(\frac{2}{\Delta t} v+\frac{1}{2}\left(a_{x}\left(A_{y} \bar{u} D_{x} v\right)+d_{x}\left(A_{y} \bar{u} A_{x} v\right)\right)+\frac{1}{2}\left(\bar{v} D_{y}\left(a_{y} v\right)+A_{y}\left(d_{y}(v \bar{v})\right)\right)\right) \\
& =-D_{y} p+\eta \Delta_{h} v-A_{y} \bar{\phi} D_{y} \mu \\
& \left.\left\{d_{x} u+d_{y} v=0\right\}\right|_{i, j},(i, j) \neq\left(N_{x}, N_{y}\right),
\end{aligned}
$$

Copyright $@$ by SIAM. Unauthorized reproduction of this article is prohibited. 


$$
\begin{aligned}
& \frac{2}{\Delta t} \phi+d_{x}\left(A_{x} \bar{\phi} u\right)+d_{y}\left(A_{y} \bar{\phi} v\right)=M \Delta_{h} \mu \\
& \mu=2 q \overline{g(\phi)}-\gamma_{1} \Delta_{h} \phi \\
& q=\overline{g(\phi)} \phi
\end{aligned}
$$

where $u \in \mathcal{E}_{x \times \bar{y}}^{e w}, v \in \mathcal{E}_{\bar{x} \times y}^{n s}, \phi, \mu \in \mathcal{C}_{\bar{x} \times \bar{y}}$ satisfy boundary conditions (2.1)-(2.6), and $p, q \in \mathcal{C}_{x \times y}$. To prove unique solvability of linear system (5.1) with $(p, 1)_{2}=0$, we only need to prove that the homogeneous linear equation system (5.2) admits only a zero solution.

Note that $\bar{u}, \bar{v}$ satisfy boundary conditions (2.3)-(2.6), and thus $A_{y} \bar{u}, A_{x} \bar{v} \in \mathcal{V}_{x \times y}^{0}$. Combining (5.2c) and boundary conditions (2.3)-(2.6) leads to

$$
\left.\left\{d_{x} u+d_{y} v=0\right\}\right|_{N_{x}, N_{y}} \text {. }
$$

Analogous to the proof of Theorem 3.2, taking the discrete inner product of (5.2a) and $(5.2 \mathrm{~b})$ with $u$ and $v$, respectively, then adding the results, we obtain

$$
\frac{2 \rho}{\Delta t}\|\mathbf{v}\|_{2}^{2}=-\eta\|\nabla \mathbf{v}\|_{2}^{2}+\left(\mu, d_{x}\left(A_{x} \phi u\right)+d_{y}\left(A_{y} \phi v\right)\right)_{2} .
$$

Computing the discrete inner product of $(5.2 \mathrm{~d})$ with $\mu$, we obtain

$$
\frac{2}{\Delta t}(\mu, \phi)_{2}=-\left(\mu, d_{x}\left(A_{x} \phi u\right)+d_{y}\left(A_{y} \phi v\right)\right)_{2}-M\|\nabla \mu\|_{2}^{2} .
$$

Computing the discrete inner product of (5.2e) with $-\frac{2}{\Delta t} \phi$ and using (5.2f), we obtain

$$
-\frac{2}{\Delta t}(\mu, \phi)_{2}=-\frac{4}{\Delta t}\|q\|_{2}^{2}-\frac{2 \gamma_{1}}{\Delta t}\|\nabla \phi\|_{2}^{2} .
$$

Adding (5.4), (5.5), and (5.6), we deduce

$$
\frac{2 \rho}{\Delta t}\|\mathbf{v}\|_{2}^{2}+\frac{2 \gamma_{1}}{\Delta t}\|\nabla \phi\|_{2}^{2}+\frac{4}{\Delta t}\|q\|_{2}^{2}+\eta\|\nabla \mathbf{v}\|_{2}^{2}+M\|\nabla \mu\|_{2}^{2}=0
$$

which implies that

$$
u=0, \quad v=0, \quad q=0, \quad D_{x} \phi=0, \quad D_{y} \phi=0, \quad D_{x} \mu=0, \quad D_{y} \mu=0 .
$$

According to $(5.2 \mathrm{~d}),(5.2 \mathrm{e})$, and (5.8), we obtain

$$
\phi=0, \quad \mu=0 .
$$

Combining (5.2a), (5.2b), and (5.8), we have

$$
\begin{aligned}
& D_{x} p_{i+\frac{1}{2}, j}=0, i=1, \ldots, N_{x}-1, j=1, \ldots, N_{y}, \\
& D_{y} p_{i, j+\frac{1}{2}}=0, i=1, \ldots, N_{x}, j=1, \ldots, N_{y}-1,
\end{aligned}
$$

which imply

$$
p_{i, j}=p_{1,1} \forall i=1, \ldots, N_{x}, j=1, \ldots, N_{y} .
$$

Noticing the solvability condition $(p, 1)_{2}=0$, we obtain

$$
p=0 .
$$

This completes the proof. 
5.2. Unique solvability for the decoupled system. To prove the unique solvability of the fully discrete scheme given in (4.8)-(4.11), we only need to prove that the three linear systems (4.8), (4.10), and (4.11) are uniquely solvable.

Theorem 5.2. For $\rho, M, \gamma_{1}, \Delta t>0$, linear system (4.8) is uniquely solvable.

Proof. First, we note that the number of equations in linear system (4.8) is equal to the number of unknowns. We then consider the following homogeneous linear equations:

$$
\left\{\begin{array}{l}
\frac{1}{\Delta t} \phi-\frac{\Delta t}{\rho} d_{x}\left(\left(A_{x} \phi^{n}\right)^{2} D_{x} \mu\right)-\frac{\Delta t}{\rho} d_{y}\left(\left(A_{y} \phi^{n}\right)^{2} D_{y} \mu\right)=M \Delta_{h} \mu \\
\mu=2 q g\left(\phi^{n}\right)-\gamma_{1} \Delta_{h} \phi \\
q=g\left(\phi^{n}\right) \phi
\end{array}\right.
$$

where $\phi, \mu \in \mathcal{C}_{\bar{x} \times \bar{y}}$ satisfy boundary conditions (2.1)-(2.2) and $q \in \mathcal{C}_{x \times y}$. To prove the unique solvability of linear system (4.8), we only need to prove that the homogeneous linear equations given in (5.14) have only a zero solution.

Due to the fact that $\phi, \mu$ satisfy boundary conditions (2.1) and (2.2), we have

$$
D_{x} \phi, D_{x} \mu \in \mathcal{E}_{x \times y}^{e w 0}, \quad D_{y} \phi, D_{y} \mu \in \mathcal{E}_{x \times y}^{n s 0} .
$$

Computing the discrete inner product of the first equation of (5.14) with $\mu$, we obtain

$$
\frac{1}{\Delta t}(\phi, \mu)_{2}+\frac{\Delta t}{\rho}\left(\left\|A_{x} \phi^{n} D_{x} \mu\right\|_{e w}^{2}+\left\|A_{y} \phi^{n} D_{y} \mu\right\|_{n s}^{2}\right)+M\|\nabla \mu\|_{2}^{2}=0
$$

Computing the discrete inner product of the second equation of (5.14) with $-\frac{1}{\Delta t} \phi$ and using the third equation of (5.14), we obtain

$$
-\frac{1}{\Delta t}(\phi, \mu)_{2}+\frac{2}{\Delta t}\|q\|_{2}^{2}+\frac{\gamma_{1}}{\Delta t}\|\nabla \phi\|_{2}^{2}=0 .
$$

Adding the above two equations leads to

$$
\frac{\Delta t}{\rho}\left(\left\|A_{x} \phi^{n} D_{x} \mu\right\|_{e w}^{2}+\left\|A_{y} \phi^{n} D_{y} \mu\right\|_{n s}^{2}\right)+M\|\nabla \mu\|_{2}^{2}+\frac{\gamma_{1}}{\Delta t}\|\nabla \phi\|_{2}^{2}+\frac{2}{\Delta t}\|q\|_{2}^{2}=0
$$

which implies

$$
D_{x} \phi=0, \quad D_{y} \phi=0, \quad D_{x} \mu=0, \quad D_{y} \mu=0, \quad q=0 .
$$

Combining (5.14) and (5.19), we obtain

$$
\phi=0, \quad \mu=0 .
$$

This completes the proof.

Theorem 5.3. For $\rho, \eta, \Delta t>0$, linear system (4.10) is uniquely solvable.

Proof. For linear system (4.10), only $\widetilde{u}^{n+1}$ and $\widetilde{v}^{n+1}$ are unknown after solving system (4.8). We consider the following homogeneous linear equation system:

$(5.21)$

$$
\left\{\begin{array}{l}
\frac{\rho}{\Delta t} \widetilde{u}+\frac{\rho}{2}\left(u^{n} D_{x}\left(a_{x} \widetilde{u}\right)+A_{x}\left(d_{x}\left(\widetilde{u} u^{n}\right)\right)\right)+\frac{\rho}{2}\left(a_{y}\left(A_{x} v^{n} D_{y} \widetilde{u}\right)+d_{y}\left(A_{y} \widetilde{u} A_{x} v^{n}\right)\right)=\eta \Delta_{h} \widetilde{u}, \\
\frac{\rho}{\Delta t} \widetilde{v}+\frac{\rho}{2}\left(a_{x}\left(A_{y} u^{n} D_{x} \widetilde{v}\right)+d_{x}\left(A_{y} u^{n} A_{x} \widetilde{v}\right)\right)+\frac{\rho}{2}\left(v^{n} D_{y}\left(a_{y} \widetilde{v}\right)+A_{y}\left(d_{y}\left(\widetilde{v} v^{n}\right)\right)\right)=\eta \Delta_{h} \widetilde{v}
\end{array}\right.
$$

Copyright (c) by SIAM. Unauthorized reproduction of this article is prohibited. 
where $\widetilde{u} \in \mathcal{E}_{x \times \bar{y}}^{e w}$ and $\widetilde{v} \in \mathcal{E}_{\bar{x} \times y}^{n s}$ satisfy discrete boundary conditions (2.3)-(2.6). To prove unique solvability of linear system (4.10), we only need to prove that the homogeneous linear equation system (5.21) has only a zero solution.

Based on the proof of Theorem 4.2, we have

$$
\widetilde{u} \in \mathcal{E}_{x \times y}^{e w 0}, \quad \widetilde{v} \in \mathcal{E}_{x \times y}^{n s 0}, \quad A_{y} \widetilde{u}, A_{x} \widetilde{v}, A_{x} v^{n} D_{y} \widetilde{u}, A_{y} u^{n} D_{x} \widetilde{v} \in \mathcal{V}_{x \times y}^{0} .
$$

Taking the discrete inner product of the two equations of (5.21) with $\widetilde{u}$ and $\widetilde{v}$, respectively, then adding the results, we deduce

$$
\frac{\rho}{\Delta t}\left(\|\widetilde{u}\|_{e w}^{2}+\|\widetilde{v}\|_{n s}^{2}\right)+\eta\left(\left\|d_{x} \widetilde{u}\right\|_{2}^{2}+\left\|D_{y} \widetilde{u}\right\|_{v c}^{2}+\left\|D_{x} \widetilde{v}\right\|_{v c}^{2}+\left\|d_{y} \widetilde{v}\right\|_{2}^{2}\right)=0,
$$

which implies

$$
\widetilde{u}=0, \quad \widetilde{v}=0 .
$$

This completes the proof.

TheOREM 5.4. For $\rho, \Delta t>0$, linear system (4.11) with the solvability condition $\left(p^{n+1}, 1\right)_{2}=0$ is uniquely solvable.

Proof. Analogous to the proof of Theorem (5.3), we consider the following homogeneous linear equations:

$$
\left\{\begin{array}{l}
\left.\left\{\frac{\rho}{\Delta t} u+D_{x} p=0\right\}\right|_{i+\frac{1}{2}, j}, i=0, \ldots, N_{x}, j=0, \ldots, N_{y}+1 \\
\left.\left\{\frac{\rho}{\Delta t} v+D_{y} p=0\right\}\right|_{i, j+\frac{1}{2}}, i=0, \ldots, N_{x}+1, j=0, \ldots, N_{y} \\
\left.\left\{d_{x} u+d_{y} v=0\right\}\right|_{i, j}, i=1, \ldots, N_{x}, j=1, \ldots, N_{y}, \text { and }(i, j) \neq\left(N_{x}, N_{y}\right) \\
(p, 1)_{2}=0, \\
p_{0, j}=p_{1, j}, \quad p_{N_{x}, j}=p_{N_{x}+1, j}, \quad j=1,2, \ldots, N_{y} \\
p_{i, 0}=p_{i, 1}, \quad p_{i, N_{y}}=p_{i, N_{y}+1}, \quad i=0,1, \ldots, N_{x}+1
\end{array}\right.
$$

where $u \in \mathcal{E}_{x \times \bar{y}}^{e w}, v \in \mathcal{E}_{\bar{x} \times y}^{n s}$, and $p \in \mathcal{C}_{\bar{x} \times \bar{y}}$ are the unknowns. Here we only need to prove that the homogeneous linear equations given in (5.25) have only a zero solution.

Computing the discrete inner product of the first two equations of (5.25) with $u$ and $v$, respectively, then adding the results, we have

$$
\frac{\rho}{\Delta t}\left(\|u\|_{e w}^{2}+\|v\|_{n s}^{2}\right)=0
$$

which implies

$$
u=0, \quad v=0 .
$$

Combining (5.25) and (5.27) leads to

$$
p=0 .
$$

This completes the proof.

Corollary 5.1. For $\rho, \eta, M, \gamma_{1}, \Delta t>0$, the decoupled scheme given in (4.8)(4.11) with the solvability condition $\left(p^{n+1}, 1\right)_{2}=0$ is uniquely solvable.

Copyright (c) by SIAM. Unauthorized reproduction of this article is prohibited. 
6. Numerical results. In this section, we present several numerical tests to verify our theoretical results. We implement the fully discrete scheme (4.1) and scheme (4.8)-(4.11) in 2D and 3D space on a CPU/GPU hybrid architecture for high performance computing. As we have alluded to earlier, one advantage of the new, linear schemes is their simplicity to implement since at each time step only a linear system needs to be solved. To solve the linear systems efficiently, we apply a novel preconditioner [16] to the linear system resulting from the coupled linear scheme; for the decoupled scheme, the preconditioner mentioned in [58] is applied.

6.1. Time accuracy test. Here, we conduct time-step refinement tests for the fully discrete schemes given by (4.1) and (4.8)-(4.11) to demonstrate their accuracy numerically. We choose $L_{x}=L_{y}=L, L=1$ and the parameter values $M=10^{-4}$, $\gamma_{1}=10^{-2}, \gamma_{2}=10^{2}, \rho=1$. We test the time accuracy first by fixing the spatial resolution. We use the initial conditions

$$
\left.\phi(\mathbf{x})\right|_{t=0}=\frac{1}{2}\left(1+\tanh \frac{0.3 L-\sqrt{(x-0.5 L)^{2}+(y-0.5 L)^{2}}}{0.02}\right),
$$

mesh size $h_{x}=h_{y}=128$, and time step $\Delta t=10^{-2} \times \frac{1}{2^{k-1}}, k=1,2,3,4, \ldots$, and the errors are calculated as the difference between the solution of the coarse time step and that of the adjacent finer time step. The $L^{2}$ errors are summarized in Figure 6.1, where we observe approximately second order convergence in time for the coupled
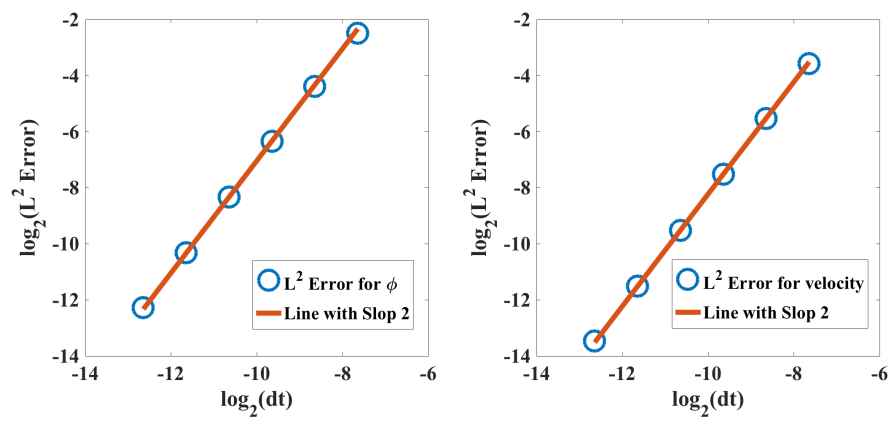

(a)
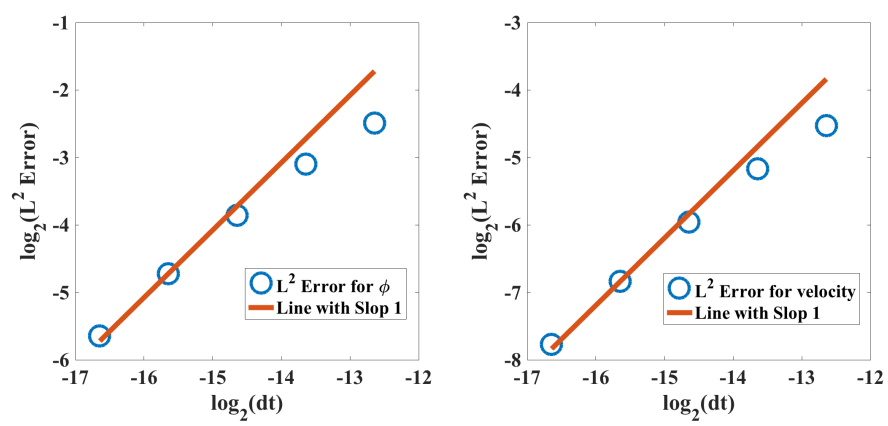

(b)

FIG. 6.1. Mesh refinement test for time accuracy. (a) Convergence test for coupled scheme (4.1), where the second order accuracy is achieved. (b) Convergence test for decoupled scheme (4.8)-(4.11), where the first order accuracy is observed. 
scheme given by (4.1), and first order convergence in time for the decoupled scheme given by (4.8)-(4.11).

6.2. Spatial accuracy test. For the spatial accuracy test, we follow the idea in [31]. We choose $N \times N$ meshes, where $N=32,96,160,224,288$, i.e., odd multiples of 32 , such that there are overlapping numerical solutions at the positions of the coarse meshes. We choose the time step as $\Delta t=10^{-5}$ to prevent the errors in time discretization from contaminating our results. We choose the same initial conditions and parameter values as those given in the previous section. Then the errors in the $L^{2}$ norms are calculated as the difference between the solution on the coarse mesh and that on the adjacent finer mesh at the positions of the coarser mesh. The order of convergence are calculated following the formula in [31]. The results are summarized in Tables 6.1 and 6.2, where we observe approximately second order convergence in space for both coupled scheme (4.1) and decoupled scheme (4.8)-(4.11).

TABLE 6.1

Spatial convergence rate test for coupled scheme (4.1).

\begin{tabular}{c|c|c|c|c}
\hline$N$ & $L^{2}$ error of $\mathbf{v}$ & Order & $L^{2}$ error of $\phi$ & Order \\
\hline 32 & $1.7009 \mathrm{e}-1$ & & $7.0739 \mathrm{e}-1$ & \\
\hline 96 & $3.4781 \mathrm{e}-2$ & 1.12 & $6.6513 \mathrm{e}-2$ & 1.78 \\
\hline 160 & $5.6681 \mathrm{e}-3$ & 2.61 & $1.8423 \mathrm{e}-2$ & 1.88 \\
\hline 224 & $2.4996 \mathrm{e}-3$ & 1.79 & $7.6165 \mathrm{e}-3$ & 1.99 \\
\hline
\end{tabular}

TABLE 6.2

Spatial convergence rate test for decoupled scheme (4.8)-(4.11).

\begin{tabular}{c|c|c|c|c}
\hline$N$ & $L^{2}$ error of $\mathbf{v}$ & Order & $L^{2}$ error of $\phi$ & Order \\
\hline 32 & $1.1444 \mathrm{e}-1$ & & $8.9551 \mathrm{e}-1$ & \\
\hline 96 & $1.9759 \mathrm{e}-2$ & 1.11 & $6.3328 \mathrm{e}-2$ & 2.03 \\
\hline 160 & $5.5627 \mathrm{e}-3$ & 1.82 & $1.7296 \mathrm{e}-2$ & 1.92 \\
\hline 224 & $2.4699 \mathrm{e}-3$ & 1.70 & $7.1004 \mathrm{e}-3$ & 1.98 \\
\hline
\end{tabular}

6.3. Comparisons between the coupled and decoupled schemes. In this section, we conduct a numerical test to compare the two linear schemes. In particular, the calculated total energies are plotted using two schemes with the same set of parameter values and initial conditions. The numerical accuracy test results are summarized in Figure 6.2. We believe the second order coupled scheme given by (4.1) is more accurate than the decoupled scheme given by (4.8)-(4.11). However, the decoupled scheme is easier to implement. Even though both schemes are unconditionally energy stable, the decoupled scheme only has first order accuracy in time, and a larger time step can introduce noticeable numerical errors, as seen with the energy curve in Figure 6.2. We also observe that the decoupled scheme provides a larger dissipation rate in magnitude (when using large time steps), which is agreeable with our theoretical results (see Theorem 4.2 and the remark below it). This enhanced dissipation may contaminate the numerical result in a long run with a large time-step size for transient flow simulations.

In the following subsections, we will use the proposed spatial-temporal, second order, coupled linear scheme to simulate two physical phenomena to demonstrate the usefulness of the scheme.

6.4. Coarsening dynamics of two viscous polymeric solutions. In this first example, we study the coarsening dynamics of two viscous polymeric solutions 


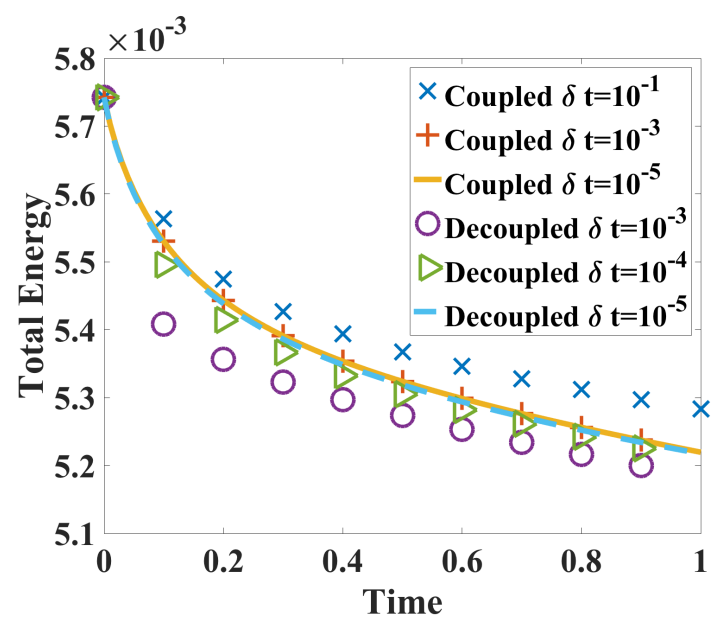

FIG. 6.2. Comparison of coupled linear scheme (4.1) and decoupled linear scheme (4.8)-(4.11). Here, we use the same parameter values as those in the previous figure to compute the total energy as a function of time. The total energies calculated using the decoupled scheme show a smaller value than the one obtained using the coupled one. The deviation decreases as the step size is reduced.

subject to different boundary conditions to investigate how boundary conditions affect the dynamics. We compare numerical results in two boundary conditions: (1) physical conditions; (2) periodic conditions. Here we use $N_{x}=N_{y}=256, L_{x}=L_{y}=2$, and $\Delta t=10^{-3}$. The initial condition is $\left.\phi(\mathbf{x})\right|_{t=0}=0.3+10^{-3} \operatorname{rand}(0,1)$ and $\left.\mathbf{v}(\mathbf{x})\right|_{t=0}=\mathbf{0}$, with $\operatorname{rand}(0,1)$ random numbers between 0 and 1 . The parameter values are $\eta=1$, $\gamma_{1}=10^{2}, \gamma_{2}=10^{-2}, M=10^{-3}, \rho=1$.

Using the same set of parameter values and initial conditions, interestingly we observe dramatically different dynamics in the computational domain. In the simulation (see Figures 6.3 and 6.4), we observe that the simulation with the physical boundary condition shows slower dynamics (i.e., energy decays more slowly) compared with the simulation with the periodic boundary condition. In fact, the velocity fields are totally different at any given time, which leads to totally different morphology in the phase during coarsening. This simulation demonstrates the dominating effect in the coarsening dynamics given by hydrodynamics and boundary conditions. The plot of the total energy decay with respect to time confirms that the total energy decays faster in the case with the periodic boundary condition than that with the physical one. So, for a mixture in a confined geometry with a zero boundary velocity, hydrodynamics can actually retard material mixing in contrast to the case with a periodic boundary condition. We believe this is the first time such a simulation evidence has been presented in the literature.

We also conduct the simulation of coarsening dynamics in 3D space. We use random initial condition $\left.\phi(\mathbf{x})\right|_{t=0}=0.3+10^{-3} \operatorname{rand}(0,1)$ for $\phi$ and zero condition for the velocity. We also use the same set of parameter values except $\eta=10^{-1}$ to facilitate the coarsening dynamics. Figure 6.5 depicts the phase coarsening dynamics in $3 \mathrm{D}$ space. The phenomena captured in $2 \mathrm{D}$ also are observed in $3 \mathrm{D}$ while the two types of boundary conditions are contrasted.

6.5. Dynamics of a rising drop. In this numerical example, we present dynamics of a lighter drop rising in a heavier fluid matrix using the Boussinesq approximation; namely, we add an extra gravity force term $-\left(\phi\left(\rho_{1}-\rho\right)+(1-\phi)\left(\rho_{2}-\rho\right)\right) g$ to 

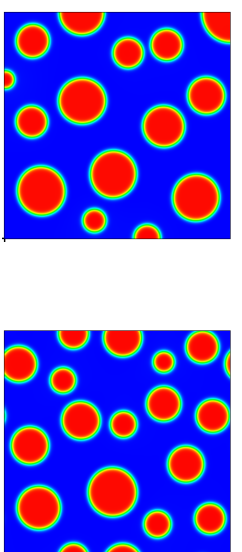

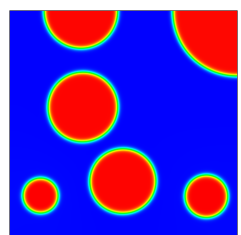

(a)
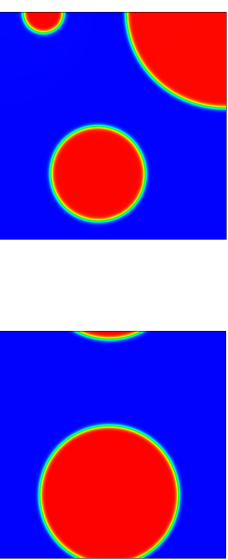

(b)

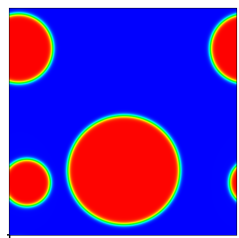

FIG. 6.3. $2 D$ coarsening dynamics. (a) Simulations subject to the physical boundary condition, where morphology of the phase variable $\phi$ is shown at $t=0,50,250,750$, respectively. (b) Simulations subject to the periodic boundary condition, where the morphology of the phase variable $\phi$ is shown at $t=0,50,250,750$, respectively. The drops are the regions in which $\phi=1$, and the background is the region where $\phi=0$.

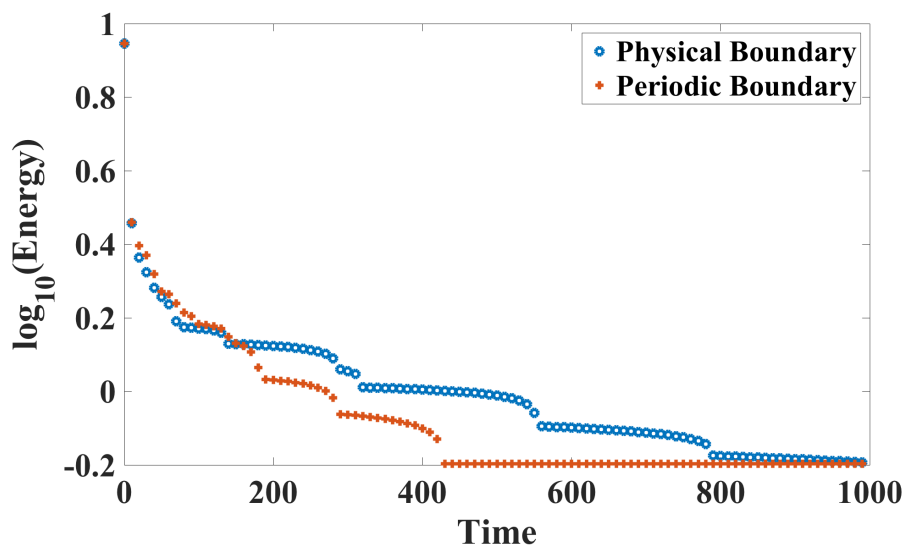

FIG. 6.4. A comparison of the total energy in the simulations depicted in Figure 6.3. The energy dissipates faster in the case with the periodic boundary condition than that with the physical boundary condition.

the momentum balance equation to approximate the upward force of buoyancy due to the density difference, where $\rho$ is the background density, $\rho_{1}, \rho_{2}$ are the actual densities for phase 1 and phase 2, and $g$ is the gravity acceleration. More details can be found in $[49,26]$. Here, we choose $L_{x}=L_{z}=1, L_{y}=2$ with mesh $N_{x}=N_{z}=128, N_{y}=256$, and initial conditions

$(6.2)$

$\left.\phi(\mathbf{x})\right|_{t=0}=\frac{1}{2}\left(1+\tanh \frac{\mathbf{x}-R}{0.02}\right), \quad R=\sqrt{\left(x-0.5 L_{x}\right)^{2}+\left(y-0.25 L_{y}\right)^{2}+\left(z-0.5 L_{z}\right)^{2}}$,

$\left.\mathbf{v}(\mathbf{x})\right|_{t=0}=\mathbf{0}$. 

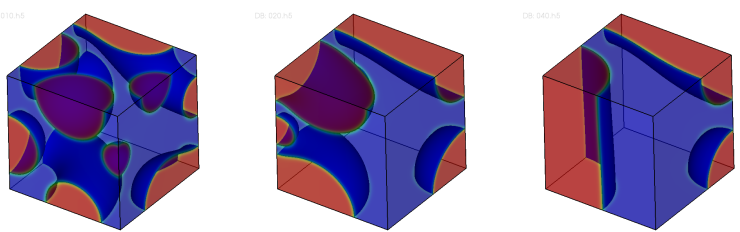

(a)
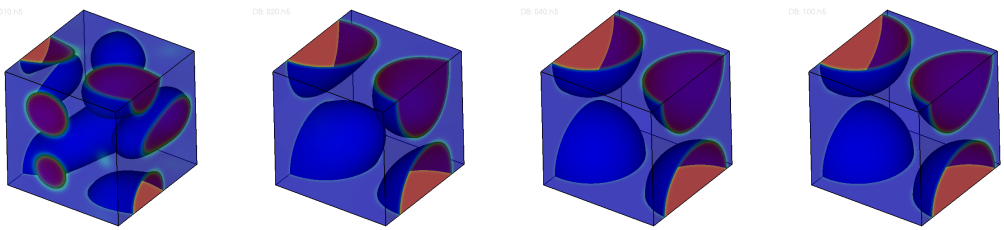

(b)

FIG. 6.5. Coarsening dynamics in 3D. (a) Coarsening dynamics with physical boundaries at $t=$ 10, 20,40,100, respectively. (b) Coarsening dynamics with periodic boundaries at $t=10,20,40,100$, respectively. Here, the red represents $\phi=1$ and blue represents $\phi=0$. (Color available online.)
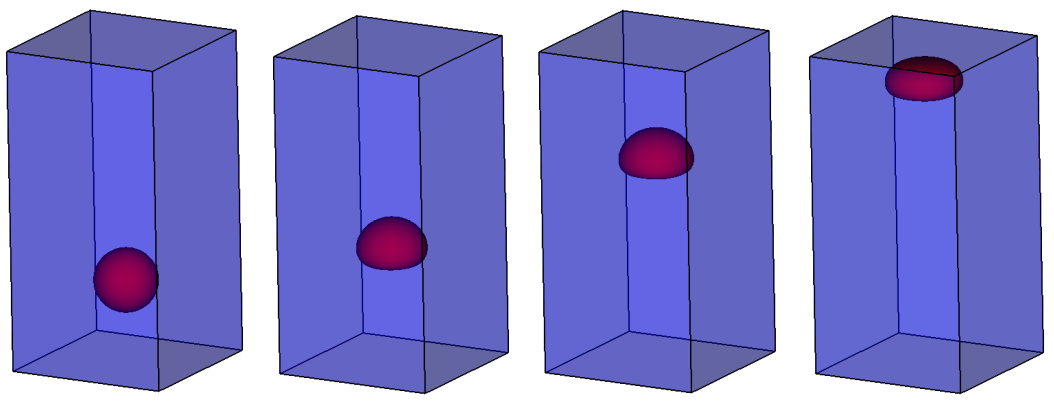

FIG. 6.6. A lighter viscous fluid drop rising in a heavier viscous fluid matrix. This figure illustrates the interface evolution at different times. The contour of $\phi=0.5$ (the interface of the fluid drop) at times $t=0,25,75,125$ is shown in red. The blue color represents the background fluid matrix. (Color available online.)

The parameters are chosen as $\rho=\rho_{2}=1, \rho_{1}=0.9, g=80, M=10^{-3}, \eta=0.1$, $\gamma_{1}=10^{-2}, \gamma_{2}=10^{2}$, and time step $\Delta t=10^{-3}$. This mimics a lighter fluid drop immersed in a heavier fluid matrix. The phase variable $\phi$ at different time slots, i.e., the evolution of the drop interface, is shown in Figure 6.6. As the initial drop profile is symmetric around the y axis and the gravity force is along the y axis, the drop should preserve axisymmetry, which is actually observed in our numerical simulation. We also observe the drop shape compressed dramatically due to the balance of the surface tension force and the buoyancy force.

To better analyze the dynamics, the velocity fields at several time slots are shown in Figure 6.7, with the red circle (the contour of $\phi=0.5$ ) representing the drop interface. We observe that a strong fluid flow is induced near the drop interface, and vortices are formed near the bottom corners of the drop. Since we enforce the no-slip boundary condition at the top $y=L_{y}$, the matrix fluid flows back downward from the top thereafter. 


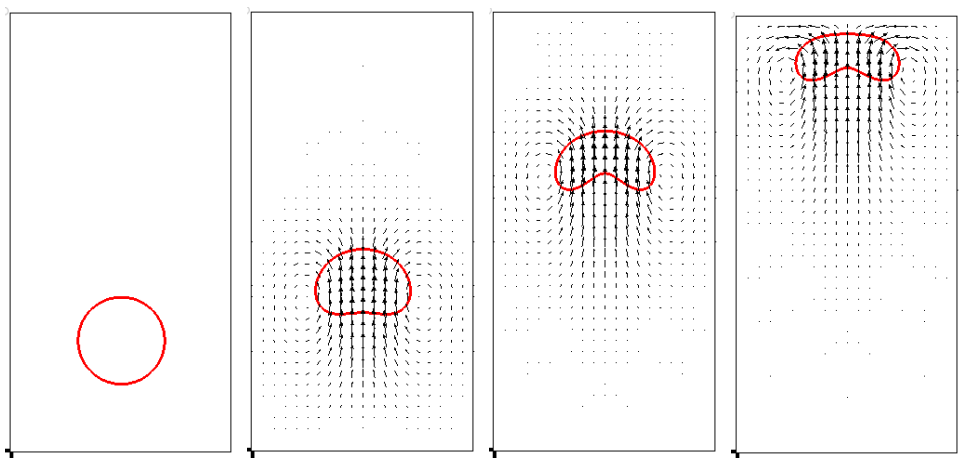

FIG. 6.7. $2 D$ slices $\left(x=0.5 L_{x}\right)$ for the simulation of a lighter fluid drop rising in a heavier fluid matrix in Figure 6.6. The contour of $\phi=0.5$ is shown in red, representing the drop interface. The black arrows represent the velocity director, with their lengths indicating the magnitude. The $2 D$ slices at time $t=0,25,75,125$ are shown, where maximum magnitudes of the velocity field are $0,1.929,1.479,0.9414$, respectively. (Color available online.)

7. Concluding remarks. We have developed two fully discrete, unconditionally energy stable schemes for the hydrodynamic phase field model of binary viscous fluid flows, consisting of a second order fully discrete, coupled linear scheme and a first order in time fully discrete, decoupled linear scheme. All the proposed schemes are unconditionally energy stable, so that they allow a relative large time step theoretically while preserving the energy stability at the fully discrete level. In the linear schemes, only linear systems need to be solved at any given time step, and their unique solvability is established rigorously. These two linear schemes have been implemented and tested numerically in 2D and 3D space. While solving the linear system at each time step, preconditioners are applied to achieve faster convergence and better stability properties. Both schemes have been verified numerically to achieve their intended order of convergence in both space and time. The advantage of the decoupled scheme is that in each time step, only an elliptic equation needs to be solved, and thus it is easy to implement and may be efficient if handled properly. The drawback is that it's only first order accurate in time, which requires smaller time steps in order to achieve the desired accuracy. On the other hand, the linear, coupled scheme gives higher accuracy (second order) in time, where a larger linear system needs to be solved at each time step. The numerical examples show that transient dynamics with respect to physical and periodic boundary conditions are indeed different at any given time, revealing the fundamental importance of applying proper boundary conditions to specific applications.

Overall, these two linear schemes are accurate and efficient, and the idea presented in this paper can be readily extended to study a broader class of multiphase hydrodynamic models for developing fully discrete, linear, unconditionally energy stable schemes.

\section{REFERENCES}

[1] H. Abels and H. Garcke, Thermodynamically consistent frame indifferent diffuse interface models for impressible two phase flows with different densities, Math. Models Methods Appl. Sci., 22 (2012), 1150013.

[2] S. Badia, F. Guillen-Gonzalez, and J. Gutierrez-Santacreu, Finite element approxima- 
tion of nematic liquid crystal flows using a saddle-point structure, J. Comput. Phys., 230 (2011), pp. 1686-1706.

[3] Y. BAO AND J. Kim, Multiphase image segmentation using a phase field model, Comput. Math. Appl., 62 (2011), pp. 737-745.

[4] A. Bertozzi, S. Esedoglu, And A. Gillette, Inpainting of binary images using the CahnHilliard equation, IEEE Trans. Image Process., 16 (2007), pp. 285-291.

[5] J. W. CAHN AND J. E. Hilliard, Free energy of a nonuniform system. I. Interfacial free energy, J. Chem. Phys., 28 (1958), pp. 258-267.

[6] L. Q. Chen And W. YAng, Computer simulation of the dynamics of a quenched system with large number of non-conserved order parameters, Phys. Rev. B, 60 (1994), pp. 15752-15756.

[7] Y. Chen And J. Shen, Efficient, adaptive energy stable schemes for the incompressible CahnHilliard Navier-Stokes phase-field models, J. Comput. Phys., 308 (2016), pp. 40-56.

[8] Q. Du, C. Liu, R. Pyham, And X. Wang, Phase field modeling of the spontaneous curvature effect in cell membranes, Comm. Pure Appl. Math., 4 (2005), pp. 537-548.

[9] Q. Du, C. Liu, AND X. WANG, Simulating the deformation of vesicle membranes under elastic bending energy in three dimensions, J. Comput. Phys., 212 (2005), pp. 757-777.

[10] W. Feng, C. Wang, S. Wise, and Z. Zhang, A Second-Order Energy Stable Backward Differentiation Formula Method for the Epitaxial Thin Film Equation with Slope Selection, preprint, https://arxiv.org/abs/1706.01943, 2017.

[11] N. Gavish, G. Hayrapetyan, K. Promislow, and L. Yang, Curvature driven flow of bilayer interfaces, Phys. D, 240 (2011), pp. 675-693.

[12] H. Gomez And T. J. R. Hughes, Provably unconditionally stable, second-order time-accurate, mixed variational methods for phase-field models, J. Comput. Phys., 230 (2011), pp. 53105327.

[13] Y. Gong, X. LIU, AND Q. WANG, Fully discretized energy stable schemes for hydrodynamic equations governing two-phase viscous fluid flows, J. Sci. Comput., 69 (2016), pp. 921-945.

[14] Y. Gong, J. ZhaO, AND Q. WANG, Linear second order in time energy stable schemes for hydrodynamic models of binary mixtures based on a spatially pseudospectral approximation, Adv. Comput. Math., to appear.

[15] Y. Gong, J. ZhaO, X. YAng, ANd Q. WANG, Fully discrete second-order linear schemes for hydrodynamic phase field models of binary viscous fluid flows with variable densities, SIAM J. Sci. Comput., 40 (2018), pp. B138-B167, https://doi.org/10.1137/17M1111759.

[16] B. E. GRIFfith, An accurate and efficient method for the incompressible Navier-Stokes equations using the projection method as a preconditioner, J. Comput. Phys., 228 (2009), pp. $7565-7595$.

[17] F. Guillen-Gonzalez And G. Tierra, Second order schemes and time-step adaptivity for Allen-Cahn and Cahn-Hilliard models, Comput. Math. Appl., 68 (2014), pp. 821-846.

[18] J. Guo, C. Wang, S. Wise, And X. YuE, An $H^{2}$ convergence of a second-order convex-splitting, finite difference scheme for the three-dimensional Cahn-Hilliard equation, Commun. Math. Sci., 14 (2015), pp. 489-515.

[19] Z. Guo AND P. Lin, A thermodynamically consistent phase-field model for two-phase flows with thermocapillary effects, J. Fluid Mech. 766 (2015), pp. 226-271.

[20] Z. Guo, P. Lin, J. Lowengrub, AND S. Wise, Mass conservative and energy stable finite difference methods for the quasi-incompressible Navier-Stokes-Cahn-Hilliard system: Primitive variable and projection-type schemes, Comput. Methods Appl. Mech. Engrg., 326 (2017), pp. 144-174.

[21] D. HAN, A. Brylev, X. YANG, AND Z. TAN, Numerical analysis of second order, fully discrete energy stable schemes for phase field models of two phase incompressible flows, J. Sci. Comput., 70 (2017), pp. 965-989.

[22] D. Han And X. Wang, A second order in time uniquely solvable unconditionally stable numerical schemes for Cahn-Hilliard-Navier-Stokes equation, J. Comput. Phys., 290 (2015), pp. 139-156.

[23] P. C. Hohenberg and B. I. Halperin, Theory of dynamic critical phenomena, Rev. Modern Phys., 49 (1977), pp. 435-479.

[24] H. Lee, J. Shin, AND J. Lee, First and second-order energy stable methods for the modified phase field crystal equation, Comput. Methods Appl. Mech. Engrg., 321 (2017), pp. 1-17.

[25] J. Li And Q. Wang, A class of conservative phase field models for multiphase fluid flows, J. Appl. Mech., 81 (2014), 021004.

[26] C. Liu And J. Shen, A phase field model for the mixture of two incompressible fluids and its approximation by a Fourier-spectral method, Phys. D, 179 (2003), pp. 211-228.

[27] J. Lober, F. Ziebert, And I. S. Aranson, Modeling crawling cell movement on soft engineered substrates, Soft Matter, 10 (2014), pp. 1365-1373.

Copyright $@$ by SIAM. Unauthorized reproduction of this article is prohibited. 
[28] J. Lober, F. Ziebert, And I. S. Aranson, Collisions of deformable cells leads to collective migration, Sci. Rep., 5 (2015), 9172.

[29] J. S. Lowengrub And L. Truskinovsky, Quasi incompressible Cahn-Hilliard fluids and topological transitions, Proc. Roy. Soc. A, 454 (1998), pp. 2617-2654.

[30] B. Palmieri, Y. Bresler, D. Wirtz, and M. Grant, Multiple scale model for cell migration in monolayers: Elastic mismatch between cells enhances motility, Sci. Rep., 5 (2015), 11745.

[31] P. Seeluangsawat, 3D Computational Investigation of Viscoelastic Biofilms Using GPUs, Ph.D. Thesis, 2011.

[32] D. Shao, W. Pappel, and H. Levine, Computational model for cell morphodynamics, Phys. Rev. Lett., 105 (2010), 108104.

[33] J. Shen, C. WAng, X. WAng, And S. M. Wise, Second-order convex splitting schemes for gradient flows with Ehrlich-Schwoebel type energy: Application to thin film epitaxy, SIAM J. Numer. Anal., 50 (2012), pp. 105-125, https://doi.org/10.1137/110822839.

[34] J. Shen, J. Xu, And J. Yang, A New Class of Efficient and Robust Energy Stable Schemes for Gradient Flows, preprint, https://arxiv.org/abs/1710.01331, 2017.

[35] J. Shen And X. YAng, Decoupled energy stable schemes for phase-field models of two-phase complex fluids, SIAM J. Sci. Comput., 36 (2014), pp. B122-B145, https://doi.org/10.1137/ 130921593.

[36] J. Shen And X. YAng, Decoupled, energy stable schemes for phase-field models of two-phase incompressible flows, SIAM J. Numer. Anal., 53 (2015), pp. 279-296, https://doi.org/10. $1137 / 140971154$.

[37] J. Shen, X. YANG, AND Q. WANG, Mass and volume conservation in phase field models for binary fluids, Commun. Comput. Phys., 13 (2013), pp. 1045-1065.

[38] E. Tuhung, D. Marenduzzo, and M. E. Cates, Spontaneous symmetry breaking in active droplets provides a generic route to motility, Proc. Natl. Acad. Sci. USA, 109 (2012), pp. 12381-12386.

[39] S. Wang, R. Sekerka, A. Wheeler, B. T. Murray, S. R. Coriell, R. J. Braun, and G. B. MCFADdEn, Thermodynamically-consistent phase-field models for solidification, Phys. D, 69 (1993), pp. 189-200.

[40] X. WANG AND Q. Du, Modeling and simulations of multi-component lipid membranes and open membranes via diffuse interface approaches, J. Math. Biol., 56 (2008), pp. 347-371.

[41] S. WISE, Unconditionally stable finite difference nonlinear multigrid simulation of the CahnHilliard-Hele-Shaw system of equations, J. Sci. Comput., 44 (2010), pp. 38-68.

[42] S. Wise, J. Kim, And J. Lowengrub, Solving the regularized strongly anisotropic CahnHilliard equation by an adaptive nonlinear multigrid method, J. Comput. Phys., 226 (2007), pp. $414-446$.

[43] S. Wise, J. Lowengrub, H. Frieboes, and B. Cristini, Three dimensional multispecies nonlinear tumor growth I: Model and numerical method, J. Theoret. Biol., 253 (2008), pp. 524543.

[44] X. YAng, Numerical approximations for the Cahn-Hilliard phase field model of the binary fluid-surfactant system, J. Sci. Comput., 74 (2018), pp. 1533-1553.

[45] X. YANG AND D. HAN, Linearly first- and second-order, unconditionally energy stable schemes for the phase field crystal equation, J. Comput. Phys., 333 (2017), pp. 1116-1134.

[46] X. YANG AND L. JU, Efficient linear schemes with unconditional energy stability for the phase field elastic bending energy model, J. Comput. Phys., 315 (2017), pp. 691-712.

[47] X. YANG, J. ZhaO, AND Q. WANG, Numerical approximations for the molecular beam epitaxial growth model based on the invariant energy quadratization method, J. Comput. Phys., 333 (2017), pp. 102-127.

[48] X. YAng, J. Zhao, Q. WANG, AND J. Shen, Numerical approximations for a three components Cahn-Hilliard phase-field model based on the invariant energy quadratization method, Math. Models Methods Appl. Sci., 27 (2017), pp. 1993-2023.

[49] J. ZhaO, H. LI, Q. WANG, AND X. YANG, Decoupled energy stable schemes for a phase field model of three-phase incompressible viscous fluid flow, J. Sci. Comput., 70 (2017), pp. $1367-$ 1389.

[50] J. Zhao, P. Seeluangsawat, and Q. Wang, Modeling antimicrobial tolerance and treatment of heterogeneous biofilms, Math. Biosci., 282 (2016), pp. 1-15.

[51] J. Zhao, Y. Shen, M. Happasalo, Z. J. Wang, and Q. Wang, A 3D numerical study of antimicrobial persistence in heterogeneous multi-species biofilms, J. Theoret. Biol., 392 (2016), pp. 83-98.

[52] J. ZHAO AND Q. WANG, A 3D multi-phase hydrodynamic model for cytokinesis of eukaryotic cells, Commun. Comput. Phys., 19 (2016), pp. 663-681.

[53] J. ZHAO AND Q. WANG, Modeling cytokinesis of eukaryotic cells driven by the actomyosin

Copyright $@$ by SIAM. Unauthorized reproduction of this article is prohibited. 
contractile ring, Internat. J. Numer. Methods Biomed. Engrg., 32 (2016), e02774.

[54] J. ZHAO AND Q. WANG, Three-dimensional numerical simulations of biofilm dynamics with quorum sensing in a flow cell, Bull. Math. Biol., 79 (2017), pp. 884-919.

[55] J. ZHAO, Q. WANG, AND X. YANG, Numerical approximations for a phase field dendritic crystal growth model based on invariant energy quadratization, Internat. J. Numer. Methods Engrg., 110 (2017), pp. 279-300.

[56] J. ZhaO, X. Yang, Y. Gong, AND Q. WAng, A novel linear second order unconditionally energy-stable scheme for a hydrodynamic $Q$ tensor model for liquid crystals, Comput. Methods Appl. Mech. Engrg., 318 (2017), pp. 803-825.

[57] J. ZhaO, X. Yang, Y. Gong, X. ZhaO, X. G. YAnG, J. Li, AND Q. WANG, A general strategy for numerical approximations of non-equilibrium models-part I: Thermodynamical systems, Internat. J. Numer. Analy. Model., to appear.

[58] J. Zhao, X. YAng, J. Shen, And Q. WAng, A decoupled energy stable scheme for a hydrodynamic phase field model of mixtures of nematic liquid crystals and viscous fluids, J. Comput. Phys., 305 (2016), pp. 539-556.

Copyright (c) by SIAM. Unauthorized reproduction of this article is prohibited. 